\title{
The Longest Path Problem is Polynomial on Cocomparability Graphs
}

\author{
Kyriaki Ioannidou and Stavros D. Nikolopoulos \\ Department of Computer Science, University of Ioannina \\ P.O.Box 1186, GR-45110 Ioannina, Greece \\ \{kioannid, stavros\}@cs.uoi.gr
}

\begin{abstract}
The longest path problem is the problem of finding a path of maximum length in a graph. As a generalization of the Hamiltonian path problem, it is NP-complete on general graphs and, in fact, on every class of graphs that the Hamiltonian path problem is NP-complete. Polynomial solutions for the longest path problem have recently been proposed for weighted trees, ptolemaic graphs, bipartite permutation graphs, interval graphs, and some small classes of graphs. Although the Hamiltonian path problem on cocomparability graphs was proved to be polynomial almost two decades ago, the complexity status of the longest path problem on cocomparability graphs has remained open until now; actually, the complexity status of the problem has remained open even on the smaller class of permutation graphs. In this paper, we present a polynomial-time algorithm for solving the longest path problem on the class of cocomparability graphs. Our result resolves the open question for the complexity of the problem on such graphs, and since cocomparability graphs form a superclass of both interval and permutation graphs, extends the polynomial solution of the longest path problem on interval graphs and provides polynomial solution to the class of permutation graphs.
\end{abstract}

Keywords: Longest path problem, cocomparability graphs, permutation graphs, polynomial algorithm, complexity.

\section{Introduction}

The problem of finding a path of maximum length in a graph (Longest Path Problem) generalizes the Hamiltonian path problem and thus it is NP-complete on general graphs; in fact, it is NPcomplete on every class of graphs that the Hamiltonian path problem is NP-complete. It is thus interesting to study the longest path problem on classes of graphs $\mathcal{C}$ where the Hamiltonian path problem is polynomial, since if a graph $G \in \mathcal{C}$ is not Hamiltonian, it makes sense in several applications to search for a longest path of $G$. Although the Hamiltonian path problem has been extensively studied in the past two decades, only recently did the longest path problem start receiving attention.

Additionally, recently the longest path problem has also received attention in the direction of approximation results, some of which imply that finding a longest path seems to be more difficult than deciding whether or not a graph admits a Hamiltonian path. Indeed, it has been proved that even if a graph has a Hamiltonian path, the problem of finding a path of length $n-n^{\varepsilon}$ for any $\varepsilon<1$ is NP-hard, where $n$ is the number of vertices of the graph [21]. Moreover, there is no polynomial-time constant-factor approximation algorithm for the longest path problem unless $\mathrm{P}=\mathrm{NP}[21]$. For related results see also $[12-14,29,30]$. 
The Hamiltonian path problem is known to be NP-complete in general graphs $[15,16]$, and remains NP-complete even when restricted to some small classes of graphs such as split graphs [17], chordal bipartite graphs, split strongly chordal graphs [23], directed path graphs [24], circle graphs [7], planar graphs [16], and grid graphs [20,26]. On the other hand, it admits polynomial time solutions on some known classes of graphs; such classes include interval graphs $[1,8]$, circular-arc graphs [8], biconvex graphs [2], and cocomparability graphs [9]. Note that the problem of finding a longest path on proper interval graphs is easy, since all connected proper interval graphs have a Hamiltonian path which can be computed in linear time [3].

Polynomial time solutions for the longest path problem are known only for small classes of graphs. Specifically, a linear-time algorithm for finding a longest path in a tree was proposed by Dijkstra early in 1960, a formal proof of which can be found in [5]. Recently, through a generalization of Dijkstra's algorithm for trees, Uehara and Uno [27] solved the longest path problem for weighted trees and block graphs in linear time and space, and for cacti in $O\left(n^{2}\right)$ time and space, where $n$ is the number of vertices of the input graph. Polynomial algorithms for the longest path problem have been also proposed on bipartite permutation and ptolemaic graphs having $O(n)$ and $O\left(n^{5}\right)$ time complexity, respectively [25,28]. Furthermore, Uehara and Uno in [27] solved the longest path problem on a subclass of interval graphs in $O\left(n^{3}(m+n \log n)\right)$ time, and as a corollary they showed that a longest path on threshold graphs can be found in $O(n+m)$ time and space. Recently, Ioannidou et al. [19] showed that the longest path problem has a polynomial solution on interval graphs by proposing an algorithm that runs in $O\left(n^{4}\right)$ time, answering thus the question left open in [27] concerning the complexity of the problem on interval graphs.

Although the Hamiltonian path problem on cocomparability graphs was proved to be polynomial almost two decades ago [9], the complexity status of the longest path problem on cocomparability graphs has remained open until now; actually, the complexity status of the problem has remained open even on the smaller class of permutation graphs. Note that, the hamiltonian cycle problem as well has been proved to be polynomial on permutation graphs [10] and cocomparability graphs [11].

In this paper we present a polynomial-time algorithm for solving the longest path problem on the class of cocomparability graphs, an important and well-known class of perfect graphs [17]. Thus, our result resolves the open question for the complexity of the problem on cocomparability graphs, and since cocomparability graphs form a superclass of both interval and permutation graphs, extends the polynomial solution of the longest path problem on interval graphs [19], and also provides polynomial solution to the class of permutation graphs.

The rest of this paper is organized as follows. In Section 2, we first review some properties of partial orders, comparability and cocomparability graphs and, then, introduce the notion of a normal antipath on a comparability graph, which is needed for our algorithm. In Section 3, we present our algorithm for solving the longest path problem on a cocomparability graph, and in Section 4 we prove the correctness and compute the time complexity of our algorithm. Finally, some concluding remarks follow in Section 5 .

\section{Theoretical Framework}

We consider finite undirected graphs with no loops or multiple edges. For a graph $G$, we denote its vertex and edge set by $V(G)$ and $E(G)$, respectively. An undirected edge is a pair of distinct vertices $u, v \in V(G)$, and is denoted by $u v$. We say that the vertex $u$ is adjacent to the vertex $v$ or, equivalently, the vertex $u$ sees the vertex $v$, if there is an edge $u v$ in $G$. If $u v \notin E(G)$ then we say that vertices $u$ and $v$ are antineighbors in $G$. Let $S$ be a set of vertices of a graph $G$; then, the cardinality of the set $S$ is denoted by $|S|$ and the subgraph of $G$ induced by $S$ is denoted by $G[S]$. Sometimes we denote by $G \backslash S$ the graph $G[V(G) \backslash S]$. The set $N(v)=\{u \in V(G): u v \in E(G)\}$ 
is called the neighborhood of the vertex $v \in V(G)$ in $G$, sometimes denoted by $N_{G}(v)$ for clarity reasons. The set $N[v]=N(v) \cup\{v\}$ is called the closed neighborhood of the vertex $v \in V(G)$. By $N_{\bar{G}}(v)$ we denote the set of the antineighbors of the vertex $v$ in the graph $G$.

For basic definitions in graph theory refer to $[4,17,22]$. A simple path (resp. antipath) of a graph $G$ is a sequence of distinct vertices $v_{1}, v_{2}, \ldots, v_{k}$ such that $v_{i} v_{i+1} \in E(G)$ (resp. $v_{i} v_{i+1} \notin E(G)$ ), for each $i, 1 \leq i \leq k-1$, and is denoted by $\left(v_{1}, v_{2}, \ldots, v_{k}\right)$; throughout the paper all paths and antipaths are considered to be simple. We denote by $V(P)$ the set of vertices in the path (antipath) $P$, and define the length of the path (antipath) $P$ to be the number of vertices in $P$, i.e., $|P|=|V(P)|$. We call right endpoint of a path (antipath) $P=\left(v_{1}, v_{2}, \ldots, v_{k}\right)$ the last vertex $v_{k}$ of $P$. Moreover, if $P=\left(v_{1}, v_{2}, \ldots, v_{i-1}, v_{i}, v_{i+1}, \ldots, v_{j}, v_{j+1}, v_{j+2}, \ldots, v_{k}\right)$ is a path (antipath) of a graph and $P_{0}=\left(v_{i}, v_{i+1}, \ldots, v_{j}\right)$ is a subpath (subantipath) of $P$, we shall denote the path (antipath) $P$ by $P=\left(v_{1}, v_{2}, \ldots, v_{i-1}, P_{0}, v_{j+1}, v_{j+2}, \ldots, v_{k}\right)$.

\subsection{Partial Orders and Cocomparability Graphs}

A partial order will be denoted by $\mathcal{P}=\left(V,<_{\mathcal{P}}\right)$, where $V$ is the finite ground set of elements or vertices and $<_{\mathcal{P}}$ is an irreflexive, antisymmetric, and transitive binary relation on $V$. Two elements $a, b \in V$ are comparable in $\mathcal{P}$ (denoted by $a \sim_{\mathcal{P}} b$ ) if $a<_{\mathcal{P}} b$ or $b<_{\mathcal{P}} a$; otherwise, they are said to be incomparable (denoted by $a \| b)$. An extension of a partial order $\mathcal{P}=\left(V,<_{\mathcal{P}}\right.$ ) is a partial order $L=\left(V,<_{L}\right)$ on the same ground set that extends $\mathcal{P}$, i.e., $a<_{\mathcal{P}} b \Rightarrow a<_{L} b$, for all $a, b \in V$. The dual partial order $\mathcal{P}^{d}$ of $\mathcal{P}=\left(V,<_{\mathcal{P}}\right)$ is a partial order $\mathcal{P}^{d}=\left(V,<_{\mathcal{P}^{d}}\right)$ such that for any two elements $a, b \in V, a<_{\mathcal{P}^{d}} b$ if and only if $b<_{\mathcal{P}} a$.

The graph $G$, edges of which are exactly the comparable pairs of a partial order $\mathcal{P}$ on $V(G)$, is called the comparability graph of $\mathcal{P}$, and is denoted by $G(\mathcal{P})$. The complement graph $\bar{G}$, whose edges are the incomparable pairs of $\mathcal{P}$, is called the cocomparability graph of $\mathcal{P}$, and is denoted by $\bar{G}(\mathcal{P})$. Alternatively, a graph $G$ is a cocomparability graph if its complement graph $\bar{G}$ has a transitive orientation, corresponding to the comparability relations of a partial order $\mathcal{P}_{\bar{G}}$. Note that a partial order $\mathcal{P}$ uniquely determines its comparability graph $G(\mathcal{P})$ and its cocomparability graph $\bar{G}(\mathcal{P})$, but the reverse is not true, i.e., a cocomparability graph $G$ has as many partial orders $\mathcal{P}_{\bar{G}}$ as the number of the transitive orientations of $\bar{G}$. Also, the class of cocomparability graphs is hereditary, that is if $G$ is a cocomparability graph, then every induced subgraph of $G$ is a cocomparability graph.

Let $G$ be a comparability graph, and let $\mathcal{P}_{G}$ be a partial order which corresponds to $G$. The graph $G$ can be represented by a directed covering graph with layers $H_{1}, H_{2}, \ldots, H_{h}$, in which each vertex is on the highest possible layer. That is, the maximal vertices of the partial order $\mathcal{P}_{G}$ are on the highest layer $H_{h}$, and for every vertex $v$ on layer $H_{i-1}$ there exists a vertex $u$ on layer $H_{i}$ such that $v<\mathcal{P}_{G} u$; such a layered representation of $G$ (respectively $\mathcal{P}_{G}$ ) is a called the Hasse diagram of $G$ (respectively $\mathcal{P}_{G}$ ) [9].

Let $\sigma=\left(V(G),<_{\sigma}\right)$ be a partial order on the vertices of a comparability graph $G$, such that for any two vertices $v, u \in V(G), v<_{\sigma} u$ if and only if $v \in H_{i}, u \in H_{j}$, and $i<j$; hereafter, we equivalently denote $v<_{\sigma} u$ by $u>_{\sigma} v$. For simplicity sometimes we shall write $v={ }_{\sigma} u$, for vertices $v, u \in V(G)$ which belong to the same layer $H_{i}$; we write $v \neq_{\sigma} u$ to denote that vertices $v, u \in V(G)$ belong to different layers. Also, $v \leq_{\sigma} u$ implies that either $v<_{\sigma} u$ or $v=_{\sigma} u$; again, we equivalently denote $v \leq_{\sigma} u$ by $u \geq_{\sigma} v$. Throughout the paper, such an ordering $\sigma$ is called a layered ordering of $G$. Note that, the partial order $\sigma$ is an extension of the partial order $\mathcal{P}_{G}$; in particular, it holds $v<\mathcal{P}_{G} u$ if and only if $v<_{\sigma} u$ and $v u \in E(G)$, for any two vertices $u, v \in V(G)$.

Since a comparability graph $G$ does not uniquely determine a partial order, hereafter we will represent a comparability graph $G$ by its Hasse diagram and we will denote the partial order $\left(V(G),<_{\mathcal{P}_{G}}\right)$ to which the Hasse diagram of $G$ corresponds by $\mathcal{P}_{G}$. Thus, we will say that $\mathcal{P}_{G}$ is 
the partial order which corresponds to the comparability graph $G$. Note that vertices in the Hasse diagram satisfy the following property: for any three vertices $v, u, w \in V(G)$ such that $v \in H_{i}$, $u \in H_{j}, w \in H_{k}$, and $i<j<k$ (or, equivalently, $v<_{\sigma} u<_{\sigma} w$ ), if $v u \in E(G)$ and $u w \in E(G)$, then $v w \in E(G)$.

The following definition and results were given by Damaschke et al. in [9], based on which they prove the correctness of their algorithm for finding a Hamiltonian path of a cocomparability graph; note that their algorithm uses the bump number algorithm which is presented in [18].

Definition 2.1 (Damaschke et al. [9]): Let $G$ be a comparability graph, and let $\mathcal{P}_{G}$ be the partial order which corresponds to $G$. A path $P=\left(v_{1}, v_{2}, \ldots, v_{k}\right)$ of the cocomparability graph $\bar{G}$ is monotone if $v_{i}<\mathcal{P}_{G} v_{j}$ implies $i<j$.

Lemma 2.1 (Damaschke et al. [9]): Let $G$ be a comparability graph, and let $\mathcal{P}_{G}$ be the partial order which corresponds to $G$. Let $P=\left(v_{1}, v_{2}, \ldots, v_{k}\right)$ be a Hamiltonian path of the cocomparability graph $\bar{G}$ such that $v_{1}$ is a minimal element of $\mathcal{P}_{G}$. Then there exists a monotone Hamiltonian path $P^{\prime}$ of $\bar{G}$ starting with $v_{1}$.

Theorem 2.1 (Damaschke et al. [9]): Let $G$ be a cocomparability graph. Then, G has a Hamiltonian path if and only if $G$ has a monotone Hamiltonian path.

It appears that the above two results hold not only for Hamiltonian paths of a cocomparability graph $\bar{G}$, but also for any path of $\bar{G}$. Indeed, let $P$ be a path of $\bar{G}$ and let $\overline{G^{\prime}}=\bar{G}[V(P)]$ be the subgraph of $\bar{G}$ induced by the vertices of $P$ (recall that cocomparability graphs have the hereditary property). Also, let $\mathcal{P}_{G^{\prime}}$ be the partial order which corresponds to $G^{\prime}$ such that $\mathcal{P}_{G}$ is an extension of $\mathcal{P}_{G^{\prime}}$, i.e., for any two vertices $u, v \in V(\bar{G})$, if $u<_{\mathcal{P}_{G}} v$ and $u, v \in V\left(\overline{G^{\prime}}\right)$, then $u<_{\mathcal{P}_{G^{\prime}}} v$. Then, since $P$ is a Hamiltonian path of $\overline{G^{\prime}}$, from Theorem 2.1 there exists a monotone path $P^{\prime}$ of $\overline{G^{\prime}}$ (with respect to $\mathcal{P}_{G^{\prime}}$ ) such that $V\left(P^{\prime}\right)=V(P)$. From Definition 2.1 it is easy to see that $P^{\prime}$ is also a monotone path of $\bar{G}$ (with respect to $\mathcal{P}_{G}$ ), since $\mathcal{P}_{G}$ is an extension of $\mathcal{P}_{G^{\prime}}$.

Additionally, since a path $P$ of a cocomparability graph $\bar{G}$ is an antipath of the comparability graph $G$, and since our algorithm for computing a longest path of a cocomparability graph $\bar{G}$ computes in fact a longest antipath of the comparability graph $G$, we restate the above definition and results and whenever $P$ denotes a path of a cocomparability graph $\bar{G}$, we refer to $P$ as an antipath of the comparability graph $G$.

We first restate Definition 2.1 as follows: an antipath $P=\left(v_{1}, v_{2}, \ldots, v_{k}\right)$ of a comparability graph $G$ is monotone if $v_{i}<\mathcal{P}_{G} v_{j}$ implies $i<j$, where $\mathcal{P}_{G}$ is the partial order which corresponds to $G$. We next restate Lemma 2.1 and Theorem 2.1 in a form stronger than the one stated in [9].

Lemma 2.2 Let $G$ be a comparability graph, and let $\mathcal{P}_{G}$ be the partial order which corresponds to $G$. Let $P=\left(v_{1}, v_{2}, \ldots, v_{k}\right)$ be an antipath of $G$ such that $v_{1}$ is a minimal element of $V(P)$ in $\mathcal{P}_{G}$. Then there exists a monotone antipath $P^{\prime}$ of $G$ starting with vertex $v_{1}$ such that $V\left(P^{\prime}\right)=V(P)$.

Theorem 2.2 Let $G$ be a comparability graph. If $P$ is an antipath of $G$, then there exists a monotone antipath $P^{\prime}$ of $G$ such that $V\left(P^{\prime}\right)=V(P)$.

The following lemma holds.

Lemma 2.3 Let $G$ be a comparability graph, and let $\sigma$ be the layered ordering of $G$. Let $P=$ $\left(v_{1}, v_{2}, \ldots, v_{k}\right)$ be an antipath of $G$, and let $v_{\ell} \notin V(P)$ be a vertex of $G$ such that $v_{1} \leq_{\sigma} v_{\ell}<_{\sigma} v_{k}$ and $v_{\ell} v_{k} \in E(G)$. Then there exist two consecutive vertices $v_{i-1}$ and $v_{i}$ in $P, 2 \leq i \leq k$, such that $v_{i-1} v_{\ell} \notin E(G)$ and $v_{\ell}<_{\sigma} v_{i}$. 
Proof. Let $P=\left(v_{1}, v_{2}, \ldots, v_{k}\right)$ be an antipath of $G$, and let $v_{\ell} \notin V(P)$ be a vertex of $G$ such that $v_{1} \leq_{\sigma} v_{\ell}<_{\sigma} v_{k}$ and $v_{\ell} v_{k} \in E(G)$. We first show that at least one vertex of $P$ does not see $v_{\ell}$. In the case where $v_{1}={ }_{\sigma} v_{\ell}$, then $v_{1}$ is such a vertex, i.e., $v_{1} v_{\ell} \notin E(G)$. Consider now that case where $v_{1}<_{\sigma} v_{\ell}<_{\sigma} v_{k}$, and assume that $v_{\ell} v_{i} \in E(G)$ for every vertex $v_{i} \in V(P), 1 \leq i \leq k$. Then for every vertex $v_{i} \in V(P), 1 \leq i \leq k$, it follows that $v_{\ell} \neq_{\sigma} v_{i}$, since vertices belonging to the same layer of the Hasse diagram of $G$ form an independent set. If $v_{2}<_{\sigma} v_{1}$, then obviously $v_{2}<_{\sigma} v_{\ell}$. Assume now that $v_{1}<_{\sigma} v_{2}$; recall that $v_{1}<_{\sigma} v_{\ell}$. If $v_{1}<_{\sigma} v_{\ell}<_{\sigma} v_{2}$, from the transitivity property it follows that $v_{2} v_{1} \in E(G)$, since $v_{2} v_{\ell} \in E(G)$ and $v_{\ell} v_{1} \in E(G)$; this is a contradiction to our assumption that $v_{1}$ and $v_{2}$ are consecutive in the antipath $P$. Thus, $v_{2}<_{\sigma} v_{\ell}$. Similarly, we can easily show by induction that for every pair $v_{x-1}, v_{x}$ of consecutive vertices in $P, 2 \leq x \leq k-1$, if $v_{x-1}<_{\sigma} v_{\ell}$ then $v_{x}<_{\sigma} v_{\ell}$, otherwise $v_{x-1} v_{x} \in E(G)$ due to the transitivity property. In particular, the same holds for the pair $v_{k-2}$ and $v_{k-1}$, i.e., from $v_{k-2}<_{\sigma} v_{\ell}$, we obtain $v_{k-1}<_{\sigma} v_{\ell}$. Recall that $v_{\ell}<_{\sigma} v_{k}$; thus, $v_{k-1}<_{\sigma} v_{\ell}<_{\sigma} v_{k}$, and since $v_{k} v_{\ell} \in E(G)$ and $v_{\ell} v_{k-1} \in E(G)$, from the transitivity property we obtain that $v_{k} v_{k-1} \in E(G)$. This comes to a contradiction to our assumption that $P$ is an antipath of $G$. Thus, there exists at least one vertex of $P$ which does not see $v_{\ell}$.

Let $v_{i-1}$ be the last vertex from left to right in $P$ (i.e., $i-1$ is the greatest index) such that $v_{i-1} v_{\ell} \notin E(G), 2 \leq i \leq k$. Therefore, for every index $j, i \leq j \leq k$, we have $v_{j} v_{\ell} \in E(G)$ and, thus, $v_{j} \neq_{\sigma} v_{\ell}$. If $i=k$, then $v_{k-1}, v_{k}$ is a pair of consecutive vertices in $P$ such that $v_{k-1} v_{\ell} \notin E(G)$ and $v_{\ell}<_{\sigma} v_{k}$, and the lemma holds. Assume that $2 \leq i \leq k-1$. We will show that $v_{\ell}<_{\sigma} v_{j}$ for every $j, i \leq j \leq k$. For $j=k, v_{\ell}<_{\sigma} v_{k}$ holds by assumption. Consider now the case where $i \leq j \leq k-1$. Assume that there exists a vertex $v_{p}, i \leq p \leq k-1$, such that $v_{p}<_{\sigma} v_{\ell}$; let $v_{p}$ be the last such vertex from left to right in $P$. Thus, $v_{\ell}<_{\sigma} v_{p+1}$, by the choice of $v_{p}$. Then, $v_{p}<_{\sigma} v_{\ell}<_{\sigma} v_{p+1}$, and since $v_{p+1} v_{\ell} \in E(G)$ and $v_{\ell} v_{p} \in E(G)$, we obtain that $v_{p+1} v_{p} \in E(G)$. This is a contradiction to our assumption that $v_{p}$ and $v_{p+1}$ are consecutive in the antipath $P$ of $G$. Therefore, there exists no vertex $v_{p}, i \leq p \leq k-1$, such that $v_{p}<_{\sigma} v_{\ell}$. Thus, we have shown that $v_{\ell}<_{\sigma} v_{j}$ for every $j$, $i \leq j \leq k$. In particular, $v_{\ell}<_{\sigma} v_{i}$. Therefore, the vertices $v_{i-1}$ and $v_{i}$ are a pair of consecutive vertices in $P$ such that $v_{i-1} v_{\ell} \notin E(G)$ and $v_{\ell}<_{\sigma} v_{i}$.

\subsection{Normal Antipaths on Comparability Graphs}

Our algorithm computes a longest path $P$ of a cocomparability graph $G$ by computing a specific type of antipaths of the comparability graph $\bar{G}$, which we call normal antipaths.

Definition 2.2 Let $G$ be a comparability graph, and let $\sigma$ be a layered ordering of $G$. The antipath $P=\left(v_{1}, v_{2}, \ldots, v_{k}\right)$ of $G$ is called normal, if $v_{1}$ is a leftmost (i.e., minimal) vertex of $V(P)$ in $\sigma$, and for every $i, 2 \leq i \leq k$, the vertex $v_{i}$ is a leftmost vertex of $N_{\bar{G}}\left(v_{i-1}\right) \cap\left\{v_{i}, v_{i+1}, \ldots, v_{k}\right\}$ in $\sigma$.

Note that in Definition 2.2, vertex $v_{1}$ is a leftmost (minimal) vertex of $V(P)$ in $\sigma$, and not necessarily a leftmost (minimal) vertex of $V(G)$ in $\sigma$. Based on Lemma 2.3 and Definition 2.2, we prove the following result.

Lemma 2.4 Let $G$ be a comparability graph, and let $\sigma$ be the layered ordering of $G$. Let $P=$ $\left(v_{1}, v_{2}, \ldots, v_{k}\right)$ be a normal antipath of $G$, and let $v_{\ell}$, and $v_{j}$ be two vertices of $P$ such that $v_{\ell}<_{\sigma} v_{j}$ and $v_{\ell} v_{j} \in E(G)$. Then $\ell<j$, i.e., $v_{\ell}$ appears before $v_{j}$ in $P$.

Proof. Let $P=\left(v_{1}, v_{2}, \ldots, v_{k}\right)$ be a normal antipath of a comparability graph $G$, and let $v_{\ell}$ and $v_{j}$ be two vertices of $P$ such that $v_{\ell}<_{\sigma} v_{j}$ and $v_{\ell} v_{j} \in E(G)$. Assume that $j<\ell$, i.e., $P=\left(v_{1}, \ldots, v_{j}, \ldots, v_{\ell}, \ldots, v_{k}\right)$. Since $P$ is a normal antipath, then $v_{1}$ is a leftmost vertex of $V(P)$ in $\sigma$; thus, $v_{1} \leq_{\sigma} v_{\ell}<_{\sigma} v_{j}$. Since $P^{\prime}=\left(v_{1}, v_{2}, \ldots, v_{j}\right)$ is an antipath, $v_{\ell} \notin V\left(P^{\prime}\right), v_{1} \leq_{\sigma} v_{\ell}<_{\sigma} v_{j}$, and $v_{\ell} v_{j} \in E(G)$, then from Lemma 2.3, we obtain that there exist two consecutive vertices $v_{i-1}$ and $v_{i}$ in $P^{\prime}, 2 \leq i \leq j$, such that $v_{i-1} v_{\ell} \notin E(G)$ and $v_{\ell}<_{\sigma} v_{i}$. However, this comes to a 
contradiction to our assumption that $P$ is a normal antipath, since from Definition 2.2 we obtain that $v_{\ell}$ should be the next vertex of $v_{i-1}$ in $P$, instead of $v_{i}$. Therefore, we obtain $\ell<j$.

Recall that, if $\mathcal{P}_{G}$ is the partial order corresponding to a comparability graph $G$, and $\sigma$ is the layered ordering of $G$, then $v_{\ell}<_{\mathcal{P}_{G}} v_{j}$ if and only if $v_{\ell}<_{\sigma} v_{j}$ and $v_{\ell} v_{j} \in E(G)$, for any two vertices $v_{\ell}, v_{j} \in V(G)$. Therefore, the definition of a monotone antipath can be paraphrased as follows: an antipath $P=\left(v_{1}, v_{2}, \ldots, v_{k}\right)$ of a comparability graph $G$ is monotone if $v_{\ell}<_{\sigma} v_{j}$ and $v_{\ell} v_{j} \in E(G)$ implies that $v_{\ell}$ appears before $v_{j}$ in $P$. Then, from Lemma 2.4 we obtain the following result.

Corollary 2.1 Let $G$ be a comparability graph. If $P$ is a normal antipath of $G$, then $P$ is a monotone antipath of $G$.

Note that the inverse of Corollary 2.1 is not always true; for example, see the antipath $P$ in Figure 1. In [9], for proving that for any Hamiltonian path $P$ of a cocomparability graph $\bar{G}$ there exists a monotone Hamiltonian path of $\bar{G}$, Damaschke et al. first show that there exists a path $P^{\prime}=\left(v_{1}, v_{2}, \ldots, v_{|V(\bar{G})|}\right)$ of $\bar{G}$ such that $v_{1}$ is a minimal vertex of either $\mathcal{P}_{G}$ or $\mathcal{P}_{G}^{d}$; recall that, $\mathcal{P}_{G}^{d}$ is the dual partial order of $\mathcal{P}_{G}$. Using the same arguments, we obtain the following lemma.

Lemma 2.5 Let $G$ be a comparability graph, and let $\mathcal{P}_{G}$ be the partial order which corresponds to $G$. If $P$ is an antipath of $G$, then there exists an antipath $P^{\prime}$ of $G$ such that $V\left(P^{\prime}\right)=V(P)$ which starts with a minimal vertex of $V(P)$ in $\mathcal{P}_{G}$.

Proof. Let $P=\left(v_{1}, v_{2}, \ldots, v_{k-1}, v_{k}, v_{k+1}, \ldots, v_{x}\right)$ be an antipath of a comparability graph $G$. Let $k$ be the smallest index such that $v_{k}$ is either a minimal or a maximal vertex of $V(P)$ in $\mathcal{P}_{G}^{d}$.

Case (I): Consider first the case where $v_{k}$ is a minimal vertex of $V(P)$ in $\mathcal{P}_{G}^{d}$. We apply Lemma 2.2 to antipath $P_{1}=\left(v_{k}, v_{k+1}, \ldots, v_{x}\right)$ and obtain a monotone antipath $P_{1}^{\prime}=$ $\left(v_{k}^{\prime}, \ldots, v_{x}^{\prime}\right)$ with respect to $\mathcal{P}_{G}^{d}$ such that $V\left(P_{1}^{\prime}\right)=V\left(P_{1}\right)$ and $v_{k}^{\prime}=v_{k}$. Therefore, $P_{2}=$ $\left(v_{1}, v_{2}, \ldots, v_{k-1}, v_{k}^{\prime}, \ldots, v_{x}^{\prime}\right)$ is an antipath of $G$ such that $V\left(P_{2}\right)=V(P)$. Since $\left(v_{1}, v_{2}, \ldots, v_{k-1}\right)$ contains no maximal vertex of $V(P)$ in $\mathcal{P}_{G}^{d}$ and $\left(v_{k}^{\prime}, \ldots, v_{x}^{\prime}\right)$ is a monotone antipath with respect to $\mathcal{P}_{G}^{d}$, it follows that $v_{x}^{\prime}$ is a maximal vertex of $V(P)=\left\{v_{1}, v_{2}, \ldots, v_{k-1}, v_{k}^{\prime}, \ldots, v_{x}^{\prime}\right\}$ in $\mathcal{P}_{G}^{d}$, or, equivalently, $v_{x}^{\prime}$ is a minimal vertex of $V(P)$ in $\mathcal{P}_{G}$. Thus, the reversed antipath $P^{\prime}=\left(v_{x}^{\prime}, v_{x-1}^{\prime}, \ldots, v_{k}^{\prime}, v_{k-1}, \ldots, v_{1}\right)$ of $P_{2}$ is an antipath of $G$ such that $V\left(P^{\prime}\right)=V(P)$ which starts with a minimal vertex of $V(P)$ in $\mathcal{P}_{G}$.

Case (II): Consider now the case there $v_{k}$ is a maximal vertex of $V(P)$ in $\mathcal{P}_{G}^{d}$. Thus, $v_{k}$ is a minimal vertex of $V(P)$ in $\mathcal{P}_{G}$. Following the Case $(\mathrm{I})$, we can obtain an antipath $P_{1}^{\prime}=$ $\left(v_{1}^{\prime}, v_{2}^{\prime}, \ldots, v_{x}^{\prime}\right)$ of $G$ such that $V\left(P_{1}^{\prime}\right)=V(P)$ which starts with a minimal vertex $v_{1}^{\prime}=v_{k}$ of $V(P)$ in $\mathcal{P}_{G}^{d}$. Based on Lemma 2.2, we can obtain a monotone antipath $P_{2}^{\prime}=\left(u_{1}^{\prime}, u_{2}^{\prime}, \ldots, u_{x}^{\prime}\right)$ of $G$ such that $V\left(P_{2}^{\prime}\right)=V\left(P_{1}^{\prime}\right)=V(P)$ which starts with a minimal vertex $u_{1}^{\prime}=v_{1}^{\prime}$ of $V(P)$ in $\mathcal{P}_{G}^{d}$. Since $P_{2}^{\prime}$ is a monotone antipath, $u_{x}^{\prime}$ is a maximal vertex of $V\left(P_{2}^{\prime}\right)=V(P)$ in $\mathcal{P}_{G}^{d}$, or, equivalently, $u_{x}^{\prime}$ is a minimal vertex of $V(P)$ in $\mathcal{P}_{G}$. Thus, the reversed antipath $P^{\prime}=\left(u_{x}^{\prime}, u_{x-1}^{\prime}, \ldots, u_{1}\right)$ of $P_{2}^{\prime}$ is an antipath of $G$ such that $V\left(P^{\prime}\right)=V(P)$ which starts with a minimal vertex of $V(P)$ in $\mathcal{P}_{G}$.

The following result is central for the correctness of our algorithm.

Lemma 2.6 Let $P$ be a longest antipath of a comparability graph $G$. Then, there exists a normal antipath $P^{\prime}$ of $G$ such that $V\left(P^{\prime}\right)=V(P)$.

Proof. Let $G$ be a comparability graph, $\mathcal{P}_{G}$ be the partial order that corresponds to $G, \sigma$ be the layered ordering of $G$, and let $P=\left(v_{1}, v_{2}, \ldots, v_{k}\right)$ be a longest antipath of $G$. If $k=1$, the lemma holds. Suppose that $k \geq 2$. We will prove that for every index $i, 2 \leq i \leq k$, there exists an antipath $P_{i}=\left(v_{1}^{\prime}, v_{2}^{\prime}, \ldots, v_{k}^{\prime}\right)$, such that $V\left(P_{i}\right)=V(P), v_{1}^{\prime}$ is a leftmost vertex of $V\left(P_{i}\right)$ in $\sigma$, and 
for every index $j, 2 \leq j \leq i$, the vertex $v_{j}^{\prime}$ is a leftmost vertex of $N_{\bar{G}}\left(v_{j-1}^{\prime}\right) \cap\left\{v_{j}^{\prime}, v_{j+1}^{\prime}, \ldots, v_{k}^{\prime}\right\}$ in $\sigma$. The proof will be done by induction on $i$.

From Lemma 2.5, we may assume that $v_{1}$ is a minimal vertex of $V(P)$ in $\mathcal{P}_{G}$, and then from Lemma 2.2 we may assume that $P$ is a monotone antipath of $G$. Thus, for every vertex $v_{i}$, $2 \leq i \leq k$, such that $v_{i}<_{\sigma} v_{1}$, we have $v_{i} v_{1} \notin E(G)$. If $v_{1}$ is a leftmost vertex of $V(P)$ in $\sigma$, then $P_{1}=P$. Consider now the case where $v_{1}$ is not a leftmost vertex of $V(P)$ in $\sigma$. Let $j$, $2 \leq j \leq k$, be the greatest index such that $v_{j}$ is a leftmost vertex of $V(P)$ in $\sigma$. If $v_{1} v_{j+1} \notin E(G)$ then $P_{1}=\left(v_{j}, v_{j-1}, \ldots, v_{1}, v_{j+1}, \ldots, v_{k}\right)$ is an antipath of $G$ such that $V\left(P_{1}\right)=V(P)$ and $v_{1}$ is a leftmost vertex of $V\left(P_{1}\right)$ in $\sigma$.

Consider now the case where $v_{1} v_{j+1} \in E(G)$. Since $P$ is monotone and $v_{1}$ appears in $P$ before $v_{j+1}$, we obtain that $v_{1}<_{\sigma} v_{j+1}$. Since $v_{j}<_{\sigma} v_{1}<_{\sigma} v_{j+1}, v_{j} v_{j+1} \notin E(G)$, and $v_{1} v_{j+1} \in E(G)$, from the transitivity property it follows that $v_{1} v_{j} \notin E(G)$. Therefore, by the construction of the Hasse diagram of $G$ (and, thus, of $\sigma$ ), there exists a vertex $v_{x}$ in $G$, such that $v_{x}={ }_{\sigma} v_{1}$ and $v_{j} v_{x} \in E(G)$; thus, $v_{j+1} v_{x} \notin E(G)$ due to the transitivity property. If $v_{x} \notin V(P)$, then $P^{\prime}=\left(v_{j}, v_{j-1}, \ldots, v_{1}, v_{x}, v_{j+1}, \ldots, v_{k}\right)$ is an antipath of $G$ longer than $P$. This is a contradiction to our assumption that $P$ is a longest antipath of $G$, thus, $v_{x} \in V(P)$. Since $P$ is monotone, $v_{j} v_{x} \in E(G)$, and $v_{j}<_{\sigma} v_{x}=_{\sigma} v_{1}$, it follows that $v_{j}$ appears in $P$ before $v_{x}$, i.e., $j+1 \leq x \leq k$. In fact, $j+2 \leq x \leq k$, since $v_{x}={ }_{\sigma} v_{1}<_{\sigma} v_{j+1}$. Then $P^{\prime}=\left(v_{j}, v_{j-1}, \ldots, v_{1}, v_{x}, v_{x-1}, \ldots, v_{j+1}\right)$ is an antipath of $G$ such that $V\left(P^{\prime}\right)=V(P) \backslash\left\{v_{x+1}, v_{x+2}, \ldots, v_{k}\right\}$. If $v_{j+1} v_{x+1} \notin E(G)$ then $P_{1}=\left(v_{j}, v_{j-1}, \ldots, v_{1}, v_{x}, v_{x-1}, \ldots, v_{j+1}, v_{x+1}, \ldots, v_{k}\right)$ is an antipath of $G$ such that $V\left(P_{1}\right)=V(P)$ and $v_{j}$ is a leftmost vertex of $V\left(P_{1}\right)$ in $\sigma$.

Consider now the case where $v_{j+1} v_{x+1} \in E(G)$. Since $P$ is monotone, $v_{j+1} v_{x+1} \in E(G)$ and $v_{j+1}$ appears in $P$ before $v_{x+1}$, we have that $v_{j+1}<_{\sigma} v_{x+1}$; thus, $v_{x}<_{\sigma} v_{j+1}<_{\sigma} v_{x+1}$. Since $v_{x} v_{j+1} \notin E(G)$, it follows by the construction of the Hasse diagram, that there exists a vertex $v_{y}$ in $G$ such that $v_{y}={ }_{\sigma} v_{j+1}$ and $v_{x} v_{y} \in E(G)$; thus, $v_{x+1} v_{y} \notin E(G)$ due to the transitivity property. Similarly to the above, $v_{y} \in V(P)$, since $P$ is a longest antipath of $G$. Since $P$ is monotone, $v_{x} v_{y} \in E(G)$ and $v_{x}<_{\sigma} v_{y}={ }_{\sigma} v_{j+1}$, it follows that $v_{x}$ appears in $P$ before $v_{y}$, i.e., $x+1 \leq y \leq k$ and, in fact, $x+2 \leq y \leq k$. Therefore, $P^{\prime}=\left(v_{j}, v_{j-1}, \ldots, v_{1}, v_{x}, v_{x-1}, \ldots, v_{j+1}, v_{y}, v_{y-1}, \ldots, v_{x+1}\right)$ is an antipath of $G$ such that $V\left(P^{\prime}\right)=V(P) \backslash\left\{v_{y+1}, v_{y+2}, \ldots, v_{k}\right\}$. Again, if $v_{x+1} v_{y+1} \notin E(G)$, then using the above transformation we obtain an antipath $P_{1}$. If $v_{x+1} v_{y+1} \in E(G)$, then we can repeat the above procedure until we find a pair of vertices $v_{x+1}$ and $v_{y+1}$ in $P$ such that $v_{y}<_{\sigma} v_{x+1}$, $x+2 \leq y \leq k$, and $v_{x+1} v_{y+1} \notin E(G)$.

Assume that such a pair of vertices $v_{x+1}$ and $v_{y+1}$ does not exists in $P$, i.e., $v_{y+1}$ is the last vertex $v_{k}$ of $P, v_{y}<_{\sigma} v_{x+1}, x+2 \leq y=k-1$, and $v_{x+1} v_{y+1} \in E(G)$. Therefore, $P^{\prime}=\left(v_{j}, v_{j-1}, \ldots, v_{1}, v_{x}, v_{x-1}, \ldots, v_{j+1}, v_{y}, v_{y-1}, \ldots, v_{x+1}\right)$ is an antipath of $G$ such that $V\left(P^{\prime}\right)=V(P) \backslash\left\{v_{y+1}\right\}$ and $y+1=k$. Since $P$ is monotone, $v_{x+1} v_{y+1} \in E(G)$, and $v_{x+1}$ appears in $P$ before $v_{y+1}$, it follows that $v_{x+1}<_{\sigma} v_{y+1}$; thus, $v_{y}<_{\sigma} v_{x+1}<_{\sigma} v_{y+1}$. Then, similarly to the above, it follows that $v_{y} v_{x+1} \notin E(G)$, and thus there exists a vertex $v_{\ell}$ in $G$ such that $v_{x+1}={ }_{\sigma} v_{\ell}$ and $v_{y} v_{\ell} \in E(G)$; thus $v_{\ell} v_{y+1} \notin E(G)$. Since $P$ is monotone, $v_{y}<_{\sigma} v_{\ell}$ and $v_{y} v_{\ell} \in E(G)$, it follows that if $v_{\ell} \in V(P)$, then $v_{\ell}$ appears in $P$ after $v_{y}$ and, in fact, after $v_{y+1}$, i.e., $y+1<\ell \leq k$. This comes to a contradiction to our assumption that $y+1=k$, i.e., $v_{y+1}$ is the last vertex $v_{k}$ of $P$. Thus, $v_{\ell} \notin V(P)$ and, therefore, $P^{\prime}=\left(v_{j}, v_{j-1}, \ldots, v_{1}, v_{x}, v_{x-1}, \ldots, v_{j+1}, v_{y}, v_{y-1}, \ldots, v_{x+1}, v_{\ell}, v_{y+1}\right)$ is an antipath of $G$ longer that $P$, since $y+1=k$ and, thus, $V\left(P^{\prime}\right)=V(P) \cup\left\{v_{\ell}\right\}$. This comes to a contradiction to our assumption that $P$ is a longest antipath of $G$. Therefore, there exists a pair of vertices $v_{x+1}$ and $v_{y+1}$ in $P$ such that $v_{y}<_{\sigma} v_{x+1}, x+2 \leq y \leq k$, and $v_{x+1} v_{y+1} \notin E(G)$. Then, $P_{1}=\left(v_{j}, v_{j-1}, \ldots, v_{1}, v_{x}, v_{x-1}, \ldots, v_{j+1}, v_{y}, v_{y-1}, \ldots, v_{x+1}, v_{y+1}, v_{y+2}, \ldots, v_{k}\right)$ is an antipath such that $V\left(P_{1}\right)=V(P)$ and $v_{j}$ is a leftmost vertex of $V\left(P_{1}\right)$ in $\sigma$. This completes the proof for the induction basis. 

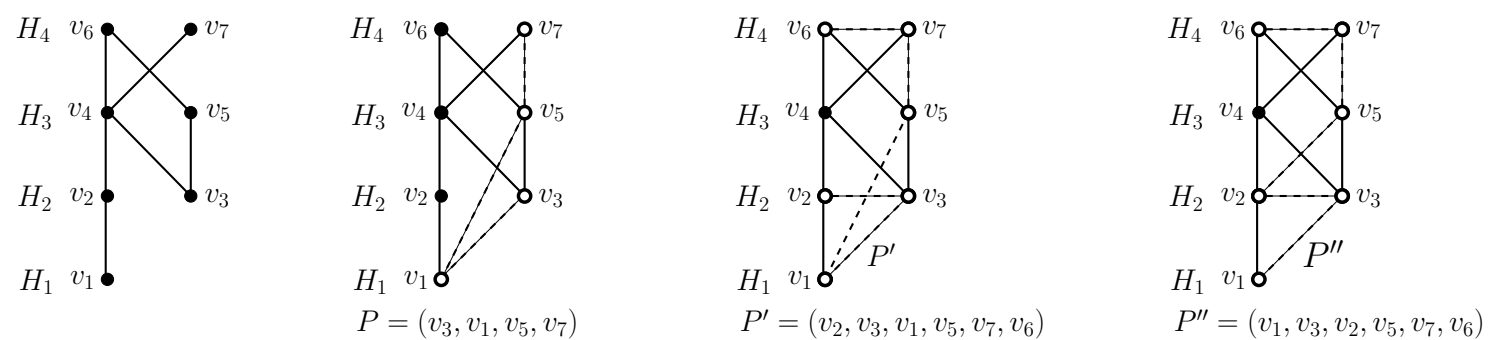

Figure 1: Illustrating a Hasse diagram of a comparability graph $G$, an antipath $P$ of $G$ which is neither normal nor longest, an antipath $P^{\prime}$ of $G$ such that $\left|P^{\prime}\right|>|P|$ which is not normal, and a normal antipath $P^{\prime \prime}$ of $G$ such that $V\left(P^{\prime \prime}\right)=V\left(P^{\prime}\right)$.

Consider now an arbitrary index $i, 2 \leq i \leq k-1$, and let $P_{i}=\left(v_{1}^{\prime}, v_{2}^{\prime}, \ldots, v_{i}^{\prime}, v_{i+1}^{\prime}, \ldots, v_{k}^{\prime}\right)$ be an antipath of $G$, such that $V\left(P_{i}\right)=V(P), v_{1}^{\prime}$ is a leftmost vertex of $V\left(P_{i}\right)$ in $\sigma$, and for every index $j, 2 \leq j \leq i$, the vertex $v_{j}^{\prime}$ is a leftmost vertex of $N_{\bar{G}}\left(v_{j-1}^{\prime}\right) \cap\left\{v_{j}^{\prime}, v_{j+1}^{\prime}, \ldots, v_{k}^{\prime}\right\}$ in $\sigma$. Therefore, the antipath $\left(v_{1}^{\prime}, v_{2}^{\prime}, \ldots, v_{i}^{\prime}\right)$ is normal. We now show that $v_{i}^{\prime}$ is a minimal vertex of $\left\{v_{i}^{\prime}, v_{i+1}^{\prime}, \ldots, v_{k}^{\prime}\right\}$ in $\mathcal{P}_{G}$. Assume otherwise that there exists a vertex $v_{x}^{\prime} \in\left\{v_{i+1}^{\prime}, v_{i+2}^{\prime}, \ldots, v_{k}^{\prime}\right\}$, such that $v_{x}^{\prime}<_{\mathcal{P}_{G}} v_{i}^{\prime}$ or, equivalently, $v_{x}^{\prime}<_{\sigma} v_{i}^{\prime}$ and $v_{x}^{\prime} v_{i}^{\prime} \in E(G)$. By the induction hypothesis, $v_{1}^{\prime}$ is a leftmost vertex of $V(P)$ in $\sigma$ and, thus, $v_{1}^{\prime} \leq_{\sigma} v_{x}^{\prime}<_{\sigma} v_{i}^{\prime}$. Since $P^{\prime}=\left(v_{1}^{\prime}, v_{2}^{\prime}, \ldots, v_{i}^{\prime}\right)$ is an antipath of $G, v_{x}^{\prime} \notin V\left(P^{\prime}\right), v_{x}^{\prime} v_{i}^{\prime} \in E(G)$, and $v_{1}^{\prime} \leq_{\sigma} v_{x}^{\prime}<_{\sigma} v_{i}^{\prime}$, from Lemma 2.3 we obtain that there exist two consecutive vertices $v_{y-1}^{\prime}$ and $v_{y}^{\prime}$ in $P^{\prime}, 2 \leq y \leq i$, such that $v_{y-1}^{\prime} v_{x}^{\prime} \notin E(G)$ and $v_{x}^{\prime}<_{\sigma} v_{y}^{\prime}$. This comes to a contradiction to our assumptions, since by the induction hypothesis $v_{y}^{\prime}$ is a leftmost vertex of $N_{\bar{G}}\left(v_{y-1}^{\prime}\right) \cap\left\{v_{y}^{\prime}, v_{y+1}^{\prime}, \ldots, v_{i}^{\prime}, \ldots, v_{x}^{\prime}, \ldots, v_{k}^{\prime}\right\}$, while $v_{x}^{\prime} \in N_{\bar{G}}\left(v_{y-1}^{\prime}\right) \cap$ $\left\{v_{y}^{\prime}, v_{y+1}^{\prime}, \ldots, v_{i}^{\prime}, \ldots, v_{x}^{\prime}, \ldots, v_{k}^{\prime}\right\}$ and $v_{x}^{\prime}<_{\sigma} v_{y}^{\prime}$. Therefore, we conclude that $v_{i}^{\prime}$ is a minimal vertex of $\left\{v_{i}^{\prime}, v_{i+1}^{\prime}, \ldots, v_{k}^{\prime}\right\}$ in $\mathcal{P}_{G}$. From Lemma 2.2, for any antipath $P$ of a comparability graph $G$ which starts with a minimal element $v$ of $V(P)$ in $\mathcal{P}_{G}$, there exists a monotone antipath $P^{\prime \prime}$ of $G$ starting with the same vertex $v$ such that $V\left(P^{\prime \prime}\right)=V(P)$. Therefore, without loss of generality we may assume that $\left\{v_{i}^{\prime}, v_{i+1}^{\prime}, \ldots, v_{k}^{\prime}\right\}$ is a monotone antipath of $G$. Therefore, by the induction hypothesis it is easy to obtain that the path $P_{i}$ is a monotone path.

If $v_{i+1}^{\prime}$ is a leftmost vertex of $N_{\bar{G}}\left(v_{i}^{\prime}\right) \cap\left\{v_{i+1}^{\prime}, v_{i+2}^{\prime}, \ldots, v_{k}^{\prime}\right\}$ in $\sigma$, then $P_{i+1}=P_{i}$. Consider now the case where $v_{i+1}$ is not a leftmost vertex of $N_{\bar{G}}\left(v_{i}^{\prime}\right) \cap\left\{v_{i+1}^{\prime}, v_{i+2}^{\prime}, \ldots, v_{k}^{\prime}\right\}$ in $\sigma$. Let $j$, $i+2 \leq j \leq k$, be the greatest index for which $v_{j}^{\prime}$ is a leftmost vertex of $N_{\bar{G}}\left(v_{i}^{\prime}\right) \cap\left\{v_{i+1}^{\prime}, v_{i+2}^{\prime}, \ldots, v_{k}^{\prime}\right\}$ in $\sigma$. Then, $P^{\prime}=\left(v_{1}^{\prime}, v_{2}^{\prime}, \ldots, v_{i}^{\prime}, v_{j}^{\prime}, v_{j-1}^{\prime}, \ldots, v_{i+1}^{\prime}\right)$ is an antipath of $G$ such that $V\left(P^{\prime}\right)=$ $V(P) \backslash\left\{v_{j+1}^{\prime}, v_{j+2}^{\prime}, \ldots, v_{k}^{\prime}\right\}$. If $v_{i+1}^{\prime} v_{j+1}^{\prime} \notin E(G)$, then $P_{i+1}=\left(v_{1}^{\prime \prime}, v_{2}^{\prime \prime}, \ldots, v_{i}^{\prime \prime}, v_{i+1}^{\prime \prime}, \ldots, v_{k}^{\prime \prime}\right)=$ $\left(v_{1}^{\prime}, v_{2}^{\prime}, \ldots, v_{i}^{\prime}, v_{j}^{\prime}, v_{j-1}^{\prime}, \ldots, v_{i+1}^{\prime}, v_{j+1}^{\prime}, v_{j+2}^{\prime}, \ldots, v_{k}^{\prime}\right)$ is an antipath of $G$ such that $V\left(P_{i+1}\right)=V(P)$, $v_{1}^{\prime \prime}$ is a leftmost vertex of $V\left(P_{i+1}\right)$ in $\sigma$, and for every index $\ell, 2 \leq \ell \leq i+1$, the vertex $v_{\ell}^{\prime \prime}$ is a leftmost vertex of $N_{\bar{G}}\left(v_{\ell-1}^{\prime \prime}\right) \cap\left\{v_{\ell}^{\prime \prime}, v_{\ell+1}^{\prime \prime}, \ldots, v_{k}^{\prime \prime}\right\}$ in $\sigma$. In the case where $v_{i+1}^{\prime} v_{j+1}^{\prime} \in E(G)$, then we repeat exactly the same procedure described in the induction basis until we find a pair of vertices $v_{x+1}^{\prime}$ and $v_{y+1}^{\prime}$ in $P$ such that $v_{y}^{\prime}<_{\sigma} v_{x+1}^{\prime}, x+2 \leq y \leq k$, and $v_{x+1}^{\prime} v_{y+1}^{\prime} \notin E(G)$; such a pair of vertices exists, as we have proven in the induction basis. Then, $P_{i+1}=$ $\left(v_{1}^{\prime \prime}, v_{2}^{\prime \prime}, \ldots, v_{i}^{\prime \prime}, v_{i+1}^{\prime \prime}, \ldots, v_{k}^{\prime \prime}\right)=\left(v_{1}^{\prime}, v_{2}^{\prime}, \ldots, v_{i}^{\prime}, v_{j}^{\prime}, v_{j-1}^{\prime}, \ldots, v_{i+1}^{\prime}, \ldots, v_{x+1}^{\prime}, v_{y+1}^{\prime}, v_{y+2}^{\prime}, \ldots, v_{k}^{\prime}\right)$ is an antipath of $G$ such that $V\left(P_{i+1}\right)=V(P), v_{1}^{\prime \prime}$ is a leftmost vertex of $V\left(P_{i+1}\right)$ in $\sigma$, and for every index $\ell, 2 \leq \ell \leq i+1$, the vertex $v_{\ell}^{\prime \prime}$ is a leftmost vertex of $N_{\bar{G}}\left(v_{\ell-1}^{\prime \prime}\right) \cap\left\{v_{\ell}^{\prime \prime}, v_{\ell+1}^{\prime \prime}, \ldots, v_{k}^{\prime \prime}\right\}$ in $\sigma$. This completes the proof for the induction step.

Thus, the antipath $P^{\prime}=P_{k}$ is a normal antipath of $G$ such that $V\left(P^{\prime}\right)=V(P)$. 
Figure 1 illustrates a Hasse diagram of a comparability graph $G$. The antipath $P=$ $\left(v_{3}, v_{1}, v_{5}, v_{7}\right)$ of $G$ is not normal, and there exists no normal antipath $\widehat{P}$ of $G$ such that $V(\widehat{P})=V(P)$; however, note that $P$ is monotone. Also, $P$ is not a longest antipath of $G$, since there exists an antipath $P^{\prime}=\left(v_{2}, v_{3}, v_{1}, v_{5}, v_{7}, v_{6}\right)$ of $G$ such that $\left|P^{\prime}\right|>|P|$. Also, $P^{\prime}$ is not a normal antipath of $G$ and there exists a normal antipath $P^{\prime \prime}=\left(v_{1}, v_{3}, v_{2}, v_{5}, v_{7}, v_{6}\right)$ of $G$ such that $V\left(P^{\prime \prime}\right)=V\left(P^{\prime}\right)$; note that it is easy to see that $P^{\prime \prime}$ is a longest antipath of $G$.

\section{The Algorithm}

Our algorithm, which we call Algorithm LP_Cocomparability, computes a longest path $P$ of a cocomparability graph $G$ by computing a longest antipath $P$ of the comparability graph $\bar{G}$.

Let $G$ be a comparability graph and let $H_{1}, H_{2}, \ldots, H_{k}$ be the layers of its Hasse diagram. For simplifying our description, we add a dummy vertex $u_{0}$ to $G$ such that $u_{0}$ belongs to a layer $H_{0}$ and $u_{0} u_{i} \in E(G)$ for every $i, 1 \leq i \leq n$; let $G^{\prime}$ be the resulting graph. Note that, $G^{\prime}$ is a comparability graph having a Hasse diagram with layers $H_{0}, H_{1}, H_{2}, \ldots, H_{k}$, and let $\sigma$ be a layered ordering of $G^{\prime}$, where $V\left(G^{\prime}\right)=\left\{u_{0}, u_{1}, u_{2}, \ldots, u_{n}\right\}$. It is easy to see that $u_{0}$ does not participate in any longest antipath $P$ of $G^{\prime}$ such that $|P| \geq 2$. In general, a longest antipath $P$ of $G^{\prime}$ which does not contain the vertex $u_{0}$ is also a longest antipath of $G$. Algorithm LP_Cocomparability computes a longest antipath of $G^{\prime}$ which is a longest antipath of the original graph $G$ as well. Hereafter, we consider comparability graphs $G$ having assumed that we have already added the dummy vertex $u_{0}$. Thus, the antipaths we compute in $G$ are also antipaths of the graph $G \backslash\left\{u_{0}\right\}$.

We next give some definitions and notations necessary for the description of the algorithm. Let $L_{j}=\left(v_{1}, v_{2}, \ldots, v_{k}\right)$ be an arbitrary ordering of the vertices $v_{1}, v_{2}, \ldots, v_{k}$. We denote by $V\left(L_{j}\right)$ the set $\left\{v_{1}, v_{2}, \ldots, v_{k}\right\}$ and by $\left|L_{j}\right|$ the cardinality of the set $V\left(L_{j}\right)$. For every vertex $v_{z} \in L_{j}$, we denote by $L_{j}\left(v_{z}\right)$ the ordering $\left(v_{1}, v_{2}, \ldots, v_{z-1}, v_{z+1}, v_{z+2}, \ldots, v_{\left|L_{j}\right|}, v_{z}\right)$, and for every index $r$, $0 \leq r \leq\left|L_{j}\right|$, we denote by $L_{j}^{r}\left(v_{z}\right)$ the ordering containing the first $r$ vertices of $L_{j}\left(v_{z}\right)$; thus:

- $L_{j}=\left(v_{1}, v_{2}, \ldots, v_{k}\right)$,

- $L_{j}\left(v_{z}\right)=\left(v_{1}, v_{2}, \ldots, v_{z-1}, v_{z+1}, v_{z+2}, \ldots, v_{\left|L_{j}\right|}, v_{z}\right)$,

- $L_{j}^{r}\left(v_{z}\right)=\left(v_{1}, v_{2}, \ldots, v_{r}\right)$ if $1 \leq r \leq z-1$,

- $L_{j}^{r}\left(v_{z}\right)=\left(v_{1}, v_{2}, \ldots, v_{z-1}, v_{z+1}, v_{z+2}, \ldots, v_{r+1}\right)$ if $z \leq r \leq\left|L_{j}\right|-1$,

- $L_{j}^{0}\left(v_{z}\right)=\emptyset$, and $L_{j}^{\left|L_{j}\right|}\left(v_{z}\right)=L_{j}\left(v_{z}\right)$.

Definition 3.1 Let $G$ be a comparability graph, let $H_{0}, H_{1}, H_{2}, \ldots, H_{k}$ be the layers of its Hasse diagram, let $V(G)=\left\{u_{0}, u_{1}, u_{2}, \ldots, u_{n}\right\}$, and let $\sigma$ be the layered ordering of $G$. For every triple $p$, $i$, and $j$, where $1 \leq i \leq j \leq k$ and $u_{p} \in H_{i-1}$, we define the graph $G\left(u_{p}, i, j\right)$ to be the subgraph $G[S]$, where $S=\left\{u_{x}: u_{x} \in H_{\ell}, i \leq \ell \leq j\right.$, and $\left.u_{p} u_{x} \in E(G)\right\}$.

In other words, graph $G\left(u_{p}, i, j\right)$ is the subgraph of $G$ induced by those vertices which belong to the layers $H_{\ell}, i \leq \ell \leq j$, and which are neighboring vertices of $u_{p} \in H_{i-1}$.

Definition 3.2 Let $L_{j}$ be an ordering of the set $H_{j} \cap V\left(G\left(u_{p}, i, j\right)\right)$. We define the graph $G_{u_{z}}^{r}\left(u_{p}, i, j\right)$, where $u_{z} \in L_{j}$ and $0 \leq r \leq\left|L_{j}\right|$, to be the subgraph $G[S]$, where $S=V\left(G\left(u_{p}, i, j-1\right)\right) \cup L_{j}^{r}\left(u_{z}\right)$ if $i<j$, and $S=L_{j}^{r}\left(u_{z}\right)$ if $i=j$.

Note that, since the dummy vertex $u_{0}$ is adjacent to every other vertex of $G$, the graph $G\left(u_{0}, 1, j\right), 1 \leq j \leq k$, is the subgraph $G[S]$ of $G$ induced by the set $S=\left\{u_{x}: u_{x} \in H_{\ell}, 1 \leq \ell \leq j\right\}$. Additionally, $G_{u_{z}}^{\left|L_{j}\right|}\left(u_{p}, i, j\right)=G\left(u_{p}, i, j\right)$, and if $i<j$, then $G_{u_{z}}^{0}\left(u_{p}, i, j\right)=G\left(u_{p}, i, j-1\right)$. 

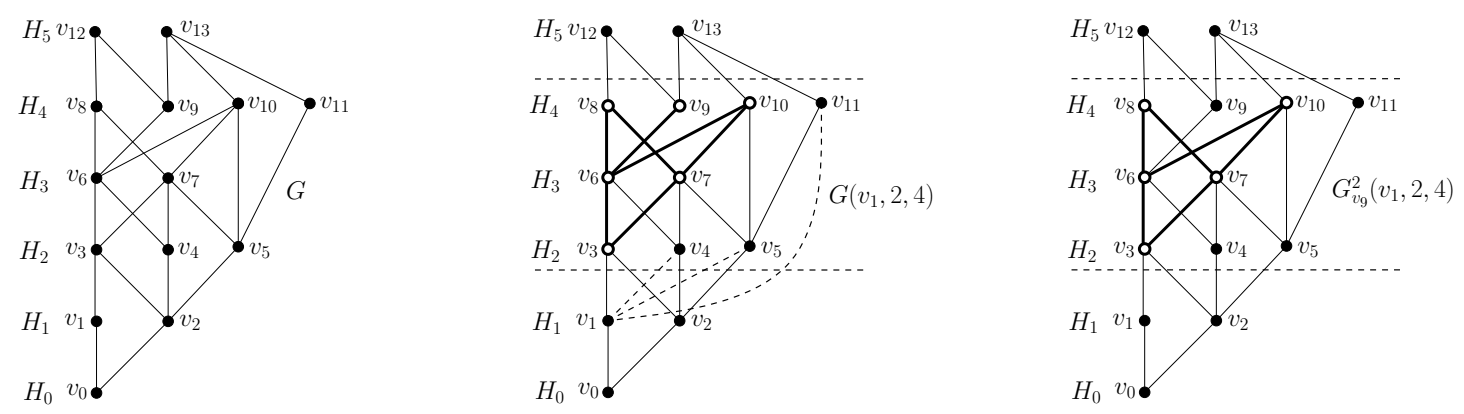

Figure 2: Illustrating a Hasse diagram of a comparability graph $G$ and the induced subgraphs $G\left(v_{1}, 2,4\right)$ and $G_{v_{9}}^{2}\left(v_{1}, 2,4\right)$ of $G$.

Figure 2 illustrates examples of the graphs defined in Definitions 3.1 and 3.2. In particular, the figure to the left illustrates a Hasse diagram of a comparability graph $G$ with layers $H_{0}, H_{1}, \ldots, H_{5}$. The figure in the middle illustrates the subgraph $G\left(v_{1}, 2,4\right)$ of $G$ induced by the vertices $\left\{v_{3}, v_{6}, v_{7}, v_{8}, v_{9}, v_{10}\right\}$. The figure to the right illustrates the subgraph $G_{v_{9}}^{2}\left(v_{1}, 2,4\right)$ of $G$, if we consider the ordering $L_{4}=\left(v_{8}, v_{9}, v_{10}\right)$ for the vertices of $H_{4} \cap V\left(G\left(v_{1}, 2,4\right)\right)$. The subgraph $G_{v_{9}}^{2}\left(v_{1}, 2,4\right)$ of $G$ is induced by the vertices $\left\{v_{3}, v_{6}, v_{7}, v_{8}, v_{10}\right\}$, and it is actually an induced subgraph of $G\left(v_{1}, 2,4\right)$.

Notation 3.1 For every vertex $u_{t} \in V\left(G_{u_{z}}^{r}\left(u_{p}, i, j\right)\right)$, if $u_{t} \in H_{j}$, then we denote by $f\left(u_{t}\right)$ the smallest index such that $f\left(u_{t}\right)<j$, for which there exists a vertex $u_{x}$ of $G_{u_{z}}^{r}\left(u_{p}, i, j\right)$ such that $u_{x} \in H_{f\left(u_{t}\right)}$ and $u_{x} u_{t} \notin E(G)$; in the case where no such index $f\left(u_{t}\right)$ exists, we set $f\left(u_{t}\right)=j$.

Notation 3.2 For every vertex $u_{y} \in V\left(G_{u_{z}}^{r}\left(u_{p}, i, j\right)\right)$ we denote by $P\left(u_{y} ; G_{u_{z}}^{r}\left(u_{p}, i, j\right)\right)$ a longest normal antipath of $G_{u_{z}}^{r}\left(u_{p}, i, j\right)$ with right endpoint the vertex $u_{y}$, and by $\ell\left(u_{y} ; G_{u_{z}}^{r}\left(u_{p}, i, j\right)\right)$ the length of $P\left(u_{y} ; G_{u_{z}}^{r}\left(u_{p}, i, j\right)\right)$.

Before describing Algorithm LP_Cocomparability in detail, we first give a high level description of our algorithm. A detailed description of Algorithm LP_Cocomparability is presented in Figures 3 and 4 .

Algorithm LP_Cocomparability. Given a comparability graph $G$ and its Hasse diagram with $H_{0}, H_{1}, \ldots, H_{k}$, our algorithm computes for every induced subgraph $G\left(u_{p}, i, j\right)$ and for every vertex $u_{y}$ of $G\left(u_{p}, i, j\right)$, the length $\ell\left(u_{y} ; G\left(u_{p}, i, j\right)\right)$ and the corresponding antipath $P\left(u_{y} ; G\left(u_{p}, i, j\right)\right)$, and outputs the maximum among the values $\left\{\ell\left(u_{y} ; G\left(u_{0}, 1, k\right)\right): u_{y} \in V\left(G\left(u_{0}, 1, k\right)\right)\right\}$ and the corresponding normal antipath $P\left(u_{y} ; G\left(u_{0}, 1, k\right)\right)$. In particular, our algorithm LP_Cocomparability works as follows:

(A) For every vertex $u_{y} \in V\left(G\left(u_{0}, 1, k\right)\right)$

compute a longest normal antipath of $G\left(u_{0}, 1, k\right)$ with right endpoint the vertex $u_{y}$, where $G\left(u_{0}, 1, k\right)=G \backslash\left\{u_{0}\right\}$.

(B) Compute the longest antipath among the $n$ antipaths computed in $(A)$.

(A.1) A longest normal antipath of $G\left(u_{0}, 1, k\right)$ with right endpoint the vertex $u_{y}$ can be computed as follows: 
- compute a longest normal antipath of $G\left(u_{p}, i, j\right)$ with right endpoint $u_{y}$, for every subgraph $G\left(u_{p}, i, j\right)$ and for every vertex $u_{y} \in V\left(G\left(u_{p}, i, j\right)\right), 1 \leq i \leq j \leq k$ and $u_{p} \in H_{i-1}$, as follows:

let $L_{j}$ be an ordering of $H_{j} \cap V\left(G\left(u_{p}, i, j\right)\right)$;

for every subgraph $G_{u_{z}}^{r}\left(u_{p}, i, j\right), 1 \leq r \leq\left|L_{j}\right|$ and $u_{z} \in L_{j}$, and for every vertex $u_{y} \in V\left(G_{u_{z}}^{r}\left(u_{p}, i, j\right)\right)$ such that $u_{y} \notin L_{j} \backslash\left\{u_{t}\right\}$ (where $u_{t}$ is the last vertex of $L_{j}^{r}\left(u_{z}\right)$ ),

compute a longest normal antipath of $G_{u_{z}}^{r}\left(u_{p}, i, j\right)$ with right endpoint the vertex $u_{y}$, where $G_{u_{z}}^{\left|L_{j}\right|}\left(u_{p}, i, j\right)=G\left(u_{p}, i, j\right), \forall u_{z} \in L_{j} ;$

(A.1.1) A longest normal antipath of $G_{u_{z}}^{r}\left(u_{p}, i, j\right)$ with right endpoint the vertex $u_{y}$ can be computed from the normal antipaths of the graph $G_{u_{z}}^{r-1}\left(u_{p}, i, j\right)$ as follows:

- compute $w_{1}=\ell\left(u_{x} ; G_{u_{z}}^{r-1}\left(u_{p}, i, j\right)\right)$ of path $P_{1}^{\prime}=P\left(u_{x} ; G_{u_{z}}^{r-1}\left(u_{p}, i, j\right)\right)$;

- compute $w_{2}=\ell\left(u_{y} ; G_{u_{z}}^{r-1}\left(u_{x}, \ell+1, j\right)\right)$ of path $P_{2}^{\prime}=P\left(u_{y} ; G_{u_{z}}^{r-1}\left(u_{x}, \ell+1, j\right)\right)$;

- if $w_{1}+w_{2}+1>\ell\left(u_{y} ; G_{u_{z}}^{r}\left(u_{p}, i, j\right)\right)$ then

$\ell\left(u_{y} ; G_{u_{z}}^{r}\left(u_{p}, i, j\right)\right) \leftarrow w_{1}+w_{2}+1$ and $P\left(u_{y} ; G_{u_{z}}^{r}\left(u_{p}, i, j\right)\right) \leftarrow\left(P_{1}^{\prime}, u_{t}, P_{2}^{\prime}\right) ;$

where $\ell \geq f\left(u_{t}\right), u_{x} \in H_{\ell} \cap V\left(G_{u_{z}}^{r-1}\left(u_{p}, i, j\right)\right)$ and $u_{x} u_{t} \notin E(G)$;

Step $(B)$ is trivial.

In Section 4.1, we prove that $P\left(u_{y} ; G\left(u_{0}, 1, k\right)\right)$ is a longest antipath of $G$. Note that, if $P$ is a longest normal antipath of $G\left(u_{p}, i, j\right)$ with right endpoint the vertex $u_{y}$, i.e., $P=P\left(u_{y} ; G\left(u_{p}, i, j\right)\right)$, then $P$ is not necessarily a longest antipath of $G\left(u_{p}, i, j\right)$. However, if $P$ is a longest antipath of $G\left(u_{p}, i, j\right)$, then from Lemma 2.6 there exists in $G\left(u_{p}, i, j\right)$ a normal antipath $P^{\prime}$ such that $V\left(P^{\prime}\right)=V(P)$; let $u_{y}$ be the right endpoint of the normal antipath $P^{\prime}$. Thus, there exists a longest normal antipath $P^{\prime}=P\left(u_{y} ; G\left(u_{p}, i, j\right)\right)$ which is also a longest antipath in $G\left(u_{p}, i, j\right)$ for some vertex $u_{y} \in V\left(G\left(u_{p}, i, j\right)\right)$.

\section{Correctness and Time Complexity}

In this section we prove the correctness of our algorithm and compute its time complexity. In particular, in Section 4.1 we show that Algorithm LP_Cocomparability correctly computes a longest normal antipath $P$ of the comparability graph $\bar{G}$, while in Section 4.2 we analyze and compute the time complexity of our algorithm.

\subsection{Correctness of Algorithm LP_Cocomparability}

Let $G$ be a comparability graph, let $H_{0}, H_{1}, H_{2}, \ldots, H_{k}$ be the layers of its Hasse diagram, and let $\sigma$ be the layered ordering of $G$. We prove the following results.

Lemma 4.1 Let $L_{j}$ be an ordering of the set $H_{j} \cap V\left(G\left(u_{p}, i, j\right)\right)$, let $P=\left(P_{1}, v_{\ell}, P_{2}\right)$ be a normal antipath of $G_{u_{z}}^{r}\left(u_{p}, i, j\right)$, and let $v_{\ell}$ be the last vertex of $L_{j}^{r}\left(u_{z}\right)$. Then, $P_{1}$ and $P_{2}$ are normal antipaths of $G_{u_{z}}^{r}\left(u_{p}, i, j\right)$.

Proof. Let $P=\left(v_{1}, v_{2}, \ldots, v_{\ell-1}, v_{\ell}, v_{\ell+1}, \ldots, v_{y}\right)$ be a normal antipath of $G_{u_{z}}^{r}\left(u_{p}, i, j\right)$. Then, from Definition 2.2, $v_{1}$ is a leftmost vertex of $V(P)$ in $\sigma$, and for every index $x, 2 \leq x \leq y$, the vertex $v_{x}$ is a leftmost vertex of $N_{\bar{G}}\left(v_{x-1}\right) \cap\left\{v_{x}, v_{x+1}, \ldots, v_{y}\right\}$ in $\sigma$. It is easy to see that $P_{1}=\left(v_{1}, v_{2}, \ldots, v_{\ell-1}\right)$ is a normal antipath of $G_{u_{z}}^{r}\left(u_{p}, i, j\right)$. Indeed, since $V\left(P_{1}\right) \subset V(P)$, then $v_{1}$ is also a leftmost vertex of $V\left(P_{1}\right)$ in $\sigma$ and, additionally, $v_{x}$ is a leftmost vertex of $N_{\bar{G}}\left(v_{x-1}\right) \cap\left\{v_{x}, v_{x+1}, \ldots, v_{\ell-1}\right\}$ in $\sigma$, for every index $x, 2 \leq x \leq \ell-1$. 


\section{Algorithm LP_Cocomparability}

Input: a comparability graph $G$ where $V(G)=\left\{u_{0}, u_{1}, u_{2}, \ldots, u_{n}\right\}$, the layers $H_{0}, H_{1}, H_{2}, \ldots, H_{k}$ of its Hasse diagram, and a layered ordering $\sigma$ of $G$.

Output: a longest normal antipath of $G$.

1. for $j=1$ to $k$

2. for $i=j$ downto 1

3. $\quad$ for every vertex $u_{p} \in H_{i-1}$

4. $\quad$ let $L_{j}$ be an ordering of $H_{j} \cap V\left(G\left(u_{p}, i, j\right)\right)$

5. $\quad$ for every vertex $u_{z} \in L_{j}$

6. $\quad$ for $r=1$ to $\left|L_{j}\right|$

7. $\quad$ let $u_{t}$ be the last vertex of $L_{j}^{r}\left(u_{z}\right)$

8. $\quad$ for every vertex $u_{y} \in V\left(G_{u_{z}}^{r}\left(u_{p}, i, j\right)\right)$ and $y \neq t \quad\{$ initialization $\}$

9. if $r=1$ then

10.

11.

12.

13.

14.

15.

16.

17.

18.

19.

20.

21.

22.

23.

24.

25.

26.

27.

28.

29.

30.

31.

32.

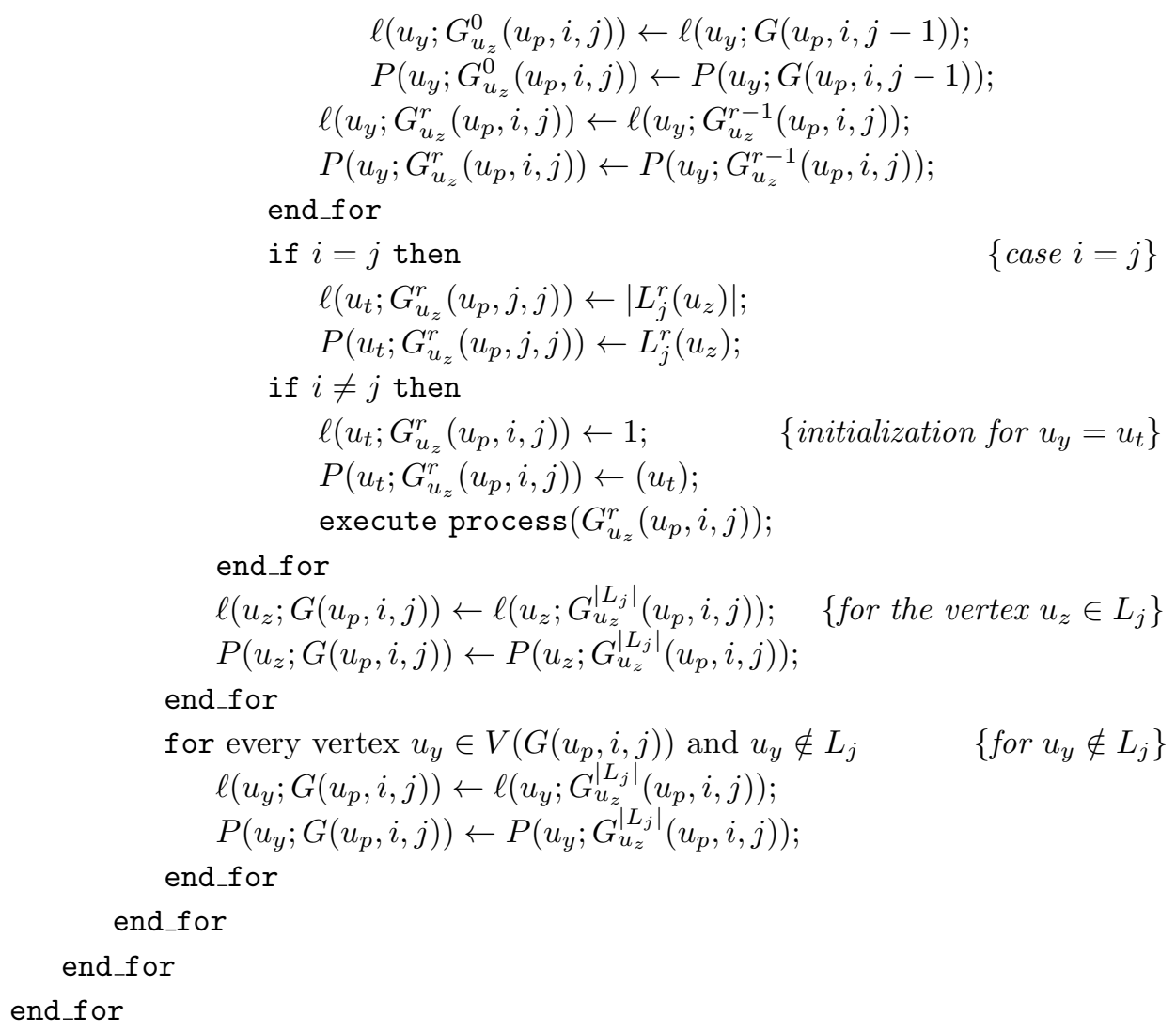

33. compute the $\max \left\{\ell\left(u_{y} ; G\left(u_{0}, 1, k\right)\right): u_{y} \in G\left(u_{0}, 1, k\right)\right\}$ and the corresponding antipath $P\left(u_{y} ; G\left(u_{0}, 1, k\right)\right)$;

Figure 3: The algorithm for finding a longest antipath of $G$. 


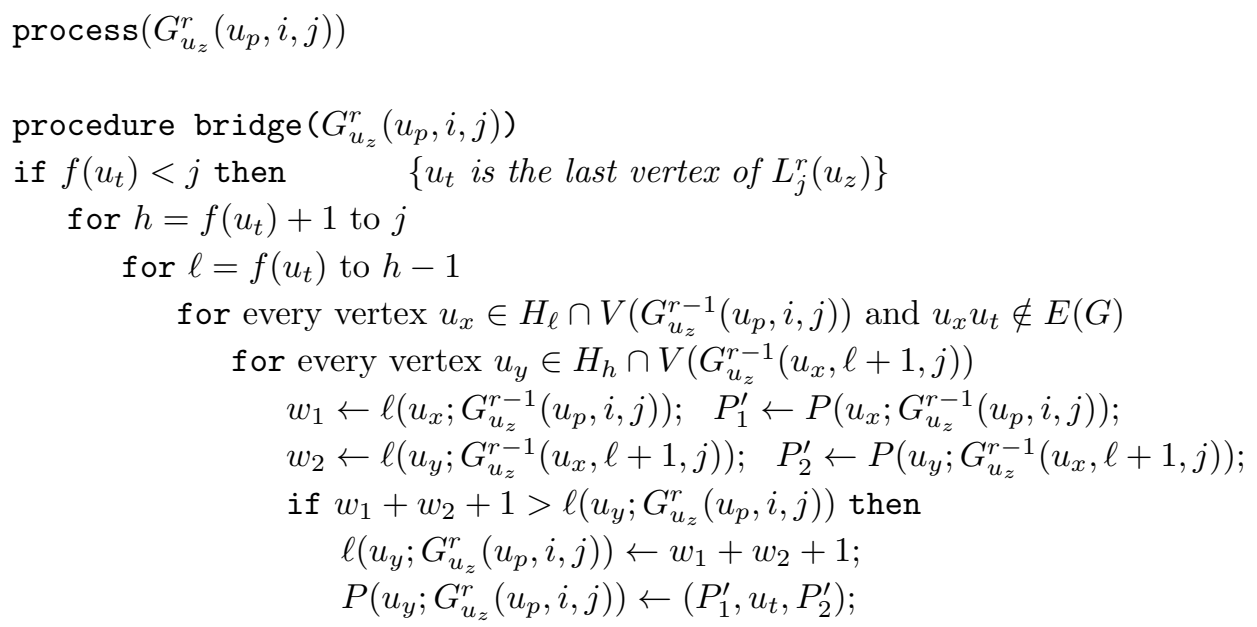

return (the value $\ell\left(u_{y} ; G_{u_{z}}^{r}\left(u_{p}, i, j\right)\right)$ and the antipath $P\left(u_{y} ; G_{u_{z}}^{r}\left(u_{p}, i, j\right)\right)$, for every vertex $u_{y} \in V\left(G_{u_{z}}^{r}\left(u_{p}, f\left(u_{t}\right)+1, j\right)\right)$ if $f\left(u_{t}\right)<j$, and for $u_{y}=u_{t}$ if $\left.f\left(u_{t}\right)=j\right)$;

Figure 4: The procedure process().

Consider now the antipath $P_{2}=\left(v_{\ell+1}, v_{\ell+2}, \ldots, v_{y}\right)$ of $G_{u_{z}}^{r}\left(u_{p}, i, j\right)$. We first prove that $v_{\ell+1}$ is a leftmost vertex of $V\left(P_{2}\right)$ in $\sigma$. By assumption $v_{\ell} \in L_{j}$, thus, $v_{x} \leq_{\sigma} v_{\ell}$ for every index $x$, $\ell+1 \leq x \leq y$. We will show that $v_{x} v_{\ell} \notin E(G)$, for every index $x, \ell+1 \leq x \leq y$. Let $v_{x}$ be a vertex of $V\left(P_{2}\right)$. Consider first the case where $v_{x}={ }_{\sigma} v_{\ell}$; then it is straightforward that $v_{x} v_{\ell} \notin E(G)$. Consider now the case where $v_{x}<_{\sigma} v_{\ell}$. Since $P$ is a normal antipath, $v_{x}<_{\sigma} v_{\ell}$, and $v_{\ell}$ appears before $v_{x}$ in $P$, from Lemma 2.4 we obtain that $v_{x} v_{\ell} \notin E(G)$. Thus, we have proved that $v_{x} v_{\ell} \notin$ $E(G)$ for every vertex $v_{x} \in V\left(P_{2}\right)$. Since $v_{\ell+1}$ is a leftmost vertex of $N_{\bar{G}}\left(v_{\ell}\right) \cap\left\{v_{\ell+1}, v_{\ell+2}, \ldots, v_{y}\right\}$ in $\sigma$, and since $N_{\bar{G}}\left(v_{\ell}\right) \cap\left\{v_{\ell+1}, v_{\ell+2}, \ldots, v_{y}\right\}=V\left(P_{2}\right)$, it follows that $v_{\ell+1}$ is a leftmost vertex of $V\left(P_{2}\right)$ in $\sigma$. Additionally, since $P$ is a normal antipath, it is straightforward that $v_{x}$ is a leftmost vertex of $N_{\bar{G}}\left(v_{x-1}\right) \cap\left\{v_{x}, v_{x+1}, \ldots, v_{y}\right\}$ in $\sigma$, for every index $x, \ell+2 \leq x \leq y$. Therefore, from Definition 2.2 it follows that $P_{2}$ is a normal antipath of $G_{u_{z}}^{r}\left(u_{p}, i, j\right)$.

The following lemma is important and shows how our algorithm LP_Cocomparability constructs normal antipaths in $G_{u_{z}}^{r}\left(u_{p}, i, j\right)$ using normal antipaths of the smaller graph $G_{u_{z}}^{r-1}\left(u_{p}, i, j\right)$.

Lemma 4.2 Let $L_{j}$ be an ordering of the set $H_{j} \cap V\left(G\left(u_{p}, i, j\right)\right)$, and let $u_{t}$ be the last vertex of $L_{j}^{r}\left(u_{z}\right)$. Let $P_{1}$ be a normal antipath of $G_{u_{z}}^{r-1}\left(u_{p}, i, j\right)$ with right endpoint a vertex $u_{x}$ such that $u_{x} \in H_{\ell}, f\left(u_{t}\right) \leq \ell \leq j-1$, and $u_{t} u_{x} \notin E(G)$. Let $P_{2}$ be a normal antipath of $G_{u_{z}}^{r-1}\left(u_{x}, \ell+1, j\right)$ with right endpoint a vertex $u_{y}$ such that $u_{y} \in H_{h}, \ell+1 \leq h \leq j$, and $V\left(P_{1}\right) \cap V\left(P_{2}\right)=\emptyset$. Then, $P=\left(P_{1}, u_{t}, P_{2}\right)$ is a normal antipath of $G_{u_{z}}^{r}\left(u_{p}, i, j\right)$ with right endpoint the vertex $u_{y}$. 
Proof. Let $P_{1}$ be a normal antipath of $G_{u_{z}}^{r-1}\left(u_{p}, i, j\right)$ with right endpoint a vertex $u_{x}$ such that $u_{x} \in H_{\ell}, f\left(u_{t}\right) \leq \ell \leq j-1$, and $u_{t} u_{x} \notin E(G)$, and let $P_{2}$ be a normal antipath of $G_{u_{z}}^{r-1}\left(u_{x}, \ell+1, j\right)$ with right endpoint a vertex $u_{y}$ such that $u_{y} \in H_{h}, \ell+1 \leq h \leq j$, and $V\left(P_{1}\right) \cap V\left(P_{2}\right)=\emptyset$. Since $u_{t} u_{x} \notin E(G), u_{x}<_{\sigma} u_{s} \leq_{\sigma} u_{t}$ and $u_{s} u_{x} \in E(G)$ for every vertex $u_{s} \in V\left(P_{2}\right)$, it follows that $u_{t} u_{s} \notin$ $E(G)$ for every vertex $u_{s} \in V\left(P_{2}\right)$. Thus, the first vertex of $P_{2}$ is an antineighbor of $u_{t}$. Therefore, since $V\left(P_{1}\right) \cap V\left(P_{2}\right)=\emptyset$, it follows that $P=\left(P_{1}, u_{t}, P_{2}\right)$ is an antipath of $G$. Additionally, since $u_{p}<_{\sigma} u_{x}<_{\sigma} u_{s}, u_{p} u_{x} \in E(G)$, and $u_{x} u_{s} \in E(G)$ for every vertex $u_{s} \in V\left(G_{u_{z}}^{r-1}\left(u_{x}, \ell+1, j\right)\right)$, from the transitivity property we obtain that $u_{p} u_{s} \in E(G)$, for every vertex $u_{s} \in V\left(P_{2}\right)$; thus, for every vertex $u_{s} \in V\left(P_{2}\right)$, we obtain $u_{s} \in V\left(G_{u_{z}}^{r-1}\left(u_{p}, i, j\right)\right)$. Therefore, since $G_{u_{z}}^{r-1}\left(u_{p}, i, j\right)$ and $G_{u z}^{r-1}\left(u_{x}, \ell+1, j\right)$ are induced subgraphs of $G_{u_{z}}^{r}\left(u_{p}, i, j\right)$, it follows that $P$ is a antipath of $G_{u_{z}}^{r}\left(u_{p}, i, j\right)$. Hereafter, in the rest of this proof $P_{1}=\left(v_{1}, v_{2}, \ldots, v_{q-1}\right), P_{2}=\left(v_{q+1}, v_{q+2}, \ldots, v_{s}\right)$, $u_{x}=v_{q-1}, u_{y}=v_{s}$, and $u_{t}=v_{q}$.

We first show that $P=\left(v_{1}, v_{2}, \ldots, v_{q}, \ldots, v_{s}\right)$ is a normal antipath. Since $v_{1}$ is a leftmost vertex of $V\left(P_{1}\right)$ in $\sigma$, it follows that $v_{1} \leq_{\sigma} u_{x}$. Furthermore, since for every vertex $v_{k} \in V\left(P_{2}\right)$ it holds $u_{x}<_{\sigma} v_{k}$, it follows that $v_{1}$ is a leftmost vertex of $V(P)$ in $\sigma$. We next show that for every $k, 2 \leq k \leq s$, the vertex $v_{k}$ is a leftmost vertex of $N_{\bar{G}}\left(v_{k-1}\right) \cap\left\{v_{k}, v_{k+1}, \ldots, v_{s}\right\}$ in $\sigma$.

Consider first the case where $2 \leq k \leq q-1$, i.e., $v_{k} \in V\left(P_{1}\right)$. Since $P_{1}$ is a normal antipath, it follows that $v_{k}$ is a leftmost vertex of $N_{\bar{G}}\left(v_{k-1}\right) \cap\left\{v_{k}, v_{k+1}, \ldots, v_{q-1}\right\}$ in $\sigma$. Consider first the case where $v_{k} \leq_{\sigma} u_{x}$. Since $u_{x}<_{\sigma} v_{k^{\prime}}$ for every vertex $v_{k^{\prime}}, q \leq k^{\prime} \leq s$, it follows that $v_{k}<_{\sigma} v_{k^{\prime}}$. Therefore, in the case where $v_{k} \leq_{\sigma} u_{x}$, we obtain that $v_{k}$ is also a leftmost vertex of $N_{\bar{G}}\left(v_{k-1}\right) \cap\left\{v_{k}, v_{k+1}, \ldots, v_{s}\right\}$ in $\sigma$. Consider now the case where $u_{x}<_{\sigma} v_{k}$. Since $v_{q}$ is a rightmost vertex of $V(P)$ is $\sigma$, it follows that $v_{k}$ is a leftmost vertex of $N_{\bar{G}}\left(v_{k-1}\right) \cap\left\{v_{k}, v_{k+1}, \ldots, v_{q-1}, v_{q}\right\}$ in $\sigma$. Now, since $u_{x}<_{\sigma} v_{k}$, and $v_{k}$ is the next vertex of $v_{k-1}$ in $P_{1}$, it follows that $v_{k-1} u_{x} \in E(G)$. Also, since $P_{1}$ is normal, $v_{k-1} u_{x} \in E(G)$, and $v_{k-1}$ appears before $u_{x}$ in $P_{1}$, from Lemma 2.4 it follows that $v_{k-1}<_{\sigma} u_{x}$. Now, since $v_{k-1}<_{\sigma} u_{x}<_{\sigma} v_{k^{\prime}}$ for every vertex $v_{k^{\prime}} \in V\left(P_{2}\right), v_{k-1} u_{x} \in E(G)$, and $u_{x} v_{k^{\prime}} \in E(G)$, from the transitivity property it follows that $v_{k-1} v_{k^{\prime}} \in E(G)$. Thus, for every vertex $v_{k^{\prime}}$ of $P_{2}$, it follows that $v_{k-1} v_{k^{\prime}} \in E(G)$. Therefore, in the case where $u_{x}<_{\sigma} v_{k}$, we obtain again that $v_{k}$ is a leftmost vertex of $N_{\bar{G}}\left(v_{k-1}\right) \cap\left\{v_{k}, v_{k+1}, \ldots, v_{s}\right\}$ in $\sigma$. Therefore, in the case where $2 \leq k \leq q-1$, we have proved that $v_{k}$ is a leftmost vertex of $N_{\bar{G}}\left(v_{k-1}\right) \cap\left\{v_{k}, v_{k+1}, \ldots, v_{s}\right\}$ in $\sigma$.

Consider now the case where $k=q$. Since $P_{1}$ is a normal antipath, and for every vertex $v_{k^{\prime}} \in V\left(P_{2}\right)$ we have that $v_{k^{\prime}} \in V\left(G_{u_{z}}^{r-1}\left(u_{x}, \ell+1, j\right)\right)$, it follows that $v_{k^{\prime}} u_{x} \in E(G)$. Therefore, $v_{q}$ is the only antineighbor of $v_{q-1}$ in $\left\{v_{q}, v_{q+1}, \ldots, v_{s}\right\}$ and, thus, $v_{q}$ is a leftmost vertex of $N_{\bar{G}}\left(v_{q-1}\right) \cap\left\{v_{q}, v_{q+1}, \ldots, v_{s}\right\}$ in $\sigma$. Now, in the case where $k=q+1$, we have that $v_{q+1}$ is a leftmost vertex of $V\left(P_{2}\right)=\left\{v_{q+1}, v_{q+2}, \ldots, v_{s}\right\}$ in $\sigma$, since $P_{2}$ is a normal antipath. Therefore, it easily follows that $v_{q+1}$ is a leftmost vertex of $N_{\bar{G}}\left(v_{q}\right) \cap\left\{v_{q+1}, v_{q+2}, \ldots, v_{s}\right\}$ in $\sigma$. Finally, in the case where $q+2 \leq k \leq s$, since $P_{2}$ is a normal antipath it directly follows that $v_{k}$ is a leftmost vertex of $N_{\bar{G}}\left(v_{k-1}\right) \cap\left\{v_{k}, v_{k+1}, \ldots, v_{s}\right\}$ in $\sigma$.

We next prove the correctness of Algorithm LP_Cocomparability. Let $G$ be a comparability graph, let $H_{0}, H_{1}, \ldots, H_{k}$ be the layers of its Hasse diagram, and let $\sigma$ be the layered ordering of $G$.

Hereafter, we distinguish the notation we use for the values computed by Algorithm LP_Cocomparability, from the notation we use for the optimum values. In particular, by $\ell\left(u_{y} ; G_{u_{z}}^{r}\left(u_{p}, i, j\right)\right)$ we denote the value computed by Algorithm LP_Cocomparability for the length of a longest normal antipath of $G_{u_{z}}^{r}\left(u_{p}, i, j\right)$ which has $u_{y}$ as its right endpoint, and by $P\left(u_{y} ; G_{u_{z}}^{r}\left(u_{p}, i, j\right)\right)$ the corresponding computed antipath. On the other hand, by $\mathcal{L}\left(u_{y} ; G_{u_{z}}^{r}\left(u_{p}, i, j\right)\right)$ we denote the optimum value of the length of a longest normal antipath of $G_{u_{z}}^{r}\left(u_{p}, i, j\right)$ which has $u_{y}$ as its right endpoint, and by $\mathcal{P}\left(u_{y} ; G_{u_{z}}^{r}\left(u_{p}, i, j\right)\right)$ the corresponding 
antipath.

Lemma 4.3 For every induced subgraph $G\left(u_{p}, i, j\right)$ of $G$, and for every vertex $u_{y} \in V\left(G\left(u_{p}, i, j\right)\right)$, the value $\ell\left(u_{y} ; G\left(u_{p}, i, j\right)\right)$ computed by Algorithm LP_Cocomparability is equal to the length of a longest normal antipath of $G\left(u_{p}, i, j\right)$ with right endpoint the vertex $u_{y}$ and, also, the corresponding computed antipath $P\left(u_{y} ; G\left(u_{p}, i, j\right)\right)$ is a longest normal antipath of $G\left(u_{p}, i, j\right)$ with right endpoint the vertex $u_{y}$.

Proof. The proof of the lemma for every subgraph $G\left(u_{p}, i, j\right), 1 \leq i \leq j \leq k$, will be done by induction on the index $j, 1 \leq j \leq k$.

Induction basis. We first prove the lemma for $j=1$, i.e., for the subgraph $G\left(u_{p}, 1,1\right)$, where $u_{p}=u_{0}$ in this case. Let $L_{1}$ be an ordering of the set $H_{1} \cap V\left(G\left(u_{p}, 1,1\right)\right)$. It is easy to see that the length of a longest normal antipath of $G\left(u_{p}, 1,1\right)$ with right endpoint a vertex $u_{z} \in L_{1}$ is equal to $\left|L_{1}\right|$, i.e., $\mathcal{L}\left(u_{z} ; G\left(u_{p}, 1,1\right)\right)=\left|L_{1}\right|$.

Let us now compare this value to the value $\ell\left(u_{z} ; G\left(u_{p}, 1,1\right)\right)$ computed by Algorithm LP_Cocomparability in this case. Since $i=j$, it easy to see that for every graph $G_{u_{z}}^{r}\left(u_{p}, 1,1\right)$, $1 \leq r \leq\left|L_{1}\right|$, Algorithm LP_Cocomparability correctly computes and sets (in lines 15-17) $\ell\left(u_{t} ; G_{u_{z}}^{r}\left(u_{p}, 1,1\right)\right)=\left|L_{1}^{r}\left(u_{z}\right)\right|$ and $P\left(u_{t} ; G_{u_{z}}^{r}\left(u_{p}, 1,1\right)\right)=L_{1}^{r}\left(u_{z}\right)$, where $u_{t}$ is the last vertex of $L_{1}^{r}\left(u_{z}\right)$. In particular, for $r=\left|L_{1}\right|$, we have $\ell\left(u_{t} ; G_{u_{z}}^{\left|L_{1}\right|}\left(u_{p}, 1,1\right)\right)=\left|L_{1}\left(u_{z}\right)\right|$ and $P\left(u_{t} ; G_{u_{z}}^{\left|L_{1}\right|}\left(u_{p}, 1,1\right)\right)=L_{1}\left(u_{z}\right)$. Moreover, Algorithm LP_Cocomparability sets (in lines 23-24) $\ell\left(u_{z} ; G\left(u_{p}, 1,1\right)\right)=\ell\left(u_{z} ; G_{u_{z}}^{\left|L_{1}\right|}\left(u_{p}, 1,1\right)\right)$ and $P\left(u_{z} ; G\left(u_{p}, 1,1\right)\right)=P\left(u_{z} ; G_{u_{z}}^{\left|L_{1}\right|}\left(u_{p}, 1,1\right)\right)$, for every vertex $u_{z}$ of $L_{1}$. Thus, Algorithm LP_Cocomparability correctly computes $\ell\left(u_{z} ; G\left(u_{p}, 1,1\right)\right)=$ $\left|L_{1}\left(u_{z}\right)\right|=\left|L_{1}\right|$ and $P\left(u_{z} ; G\left(u_{p}, 1,1\right)\right)=L_{1}\left(u_{z}\right)$, for every vertex $u_{z}$ of $L_{1}$. Therefore, $\ell\left(u_{z} ; G\left(u_{p}, 1,1\right)\right)=\mathcal{L}\left(u_{z} ; G\left(u_{p}, 1,1\right)\right)$ and $P\left(u_{z} ; G\left(u_{p}, 1,1\right)\right)=\mathcal{P}\left(u_{z} ; G\left(u_{p}, 1,1\right)\right)$, for every vertex $u_{z}$ of $L_{1}$; thus, the lemma holds for every subgraph $G_{u_{z}}^{r}\left(u_{p}, 1,1\right), 1 \leq r \leq\left|L_{1}\right|$. This proves the induction basis.

Induction hypothesis. Assume now that the lemma holds for every index $j^{\prime}, 1 \leq j^{\prime} \leq j-$ $1 \leq k-1$. That is, assume that for every induced subgraph $G\left(u_{p}, i^{\prime}, j^{\prime}\right)$ of $G, 1 \leq i^{\prime} \leq j^{\prime} \leq$ $j-1 \leq k-1$, and for every vertex $u_{y} \in V\left(G\left(u_{p}, i^{\prime}, j^{\prime}\right)\right)$, the value $\ell\left(u_{y} ; G\left(u_{p}, i^{\prime}, j^{\prime}\right)\right)$ computed by Algorithm LP_Cocomparability is equal to the length $\mathcal{L}\left(u_{y} ; G\left(u_{p}, i^{\prime}, j^{\prime}\right)\right)$ of a longest normal antipath of $G\left(u_{p}, i^{\prime}, j^{\prime}\right)$ with right endpoint the vertex $u_{y}$ and, also, the corresponding computed antipath $P\left(u_{y} ; G\left(u_{p}, i^{\prime}, j^{\prime}\right)\right)$ is a longest normal antipath of $G\left(u_{p}, i^{\prime}, j^{\prime}\right)$ with right endpoint the vertex $u_{y}$.

Induction step. We will next show that the lemma holds for $j^{\prime}=j, 1 \leq i \leq j \leq k$, i.e., for every induced subgraph $G\left(u_{p}, i, j\right)$ of $G$.

Case A. Consider first the case where $1 \leq i=j \leq k$. Let $L_{j}$ be an ordering of the set $H_{j} \cap$ $V\left(G\left(u_{p}, j, j\right)\right)$. It is easy to see that the length of a longest normal antipath of $G\left(u_{p}, j, j\right)$ with right endpoint a vertex $u_{z} \in L_{j}$ is equal to $\left|L_{j}\right|$, i.e., $\mathcal{L}\left(u_{z} ; G\left(u_{p}, j, j\right)\right)=\left|L_{j}\right|$.

Let us now compare this value to the value $\ell\left(u_{z} ; G\left(u_{p}, j, j\right)\right)$ computed by Algorithm LP_Cocomparability. Let $u_{t}$ be the last vertex of $L_{j}^{r}\left(u_{z}\right)$. We first show that for every graph $G_{u_{z}}^{r}\left(u_{p}, j, j\right), 1 \leq r \leq\left|L_{j}\right|$, Algorithm LP_Cocomparability correctly computes the values $\ell\left(u_{t} ; G_{u_{z}}^{r}\left(u_{p}, j, j\right)\right)$ and $P\left(u_{t} ; G_{u_{z}}^{r}\left(u_{p}, j, j\right)\right)$. It is easy to see that $\mathcal{L}\left(u_{t} ; G_{u_{z}}^{r}\left(u_{p}, j, j\right)\right)=\left|L_{j}^{r}\left(u_{z}\right)\right|$. In the case where $i=j$, Algorithm LP_Cocomparability correctly computes and sets (in lines 15-17) $\ell\left(u_{t} ; G_{u_{z}}^{r}\left(u_{p}, j, j\right)\right)=\left|L_{j}^{r}\left(u_{z}\right)\right|$ and $P\left(u_{t} ; G_{u_{z}}^{r}\left(u_{p}, j, j\right)\right)=L_{j}^{r}\left(u_{z}\right)$; note that for $r=\left|L_{j}\right|$, we have $\left|L_{j}^{r}\left(u_{z}\right)\right|=\left|L_{j}\right|$. Since Algorithm LP_Cocomparability computes these values for every vertex $u_{z} \in L_{j}$, i.e., for every subgraph $\left.G_{u_{z}}^{r}\left(u_{p}, j, j\right)\right)$, and since $G\left(u_{p}, j, j\right)=G_{u_{z}}^{\left|L_{j}\right|}\left(u_{p}, j, j\right)$ for any vertex $u_{z} \in L_{j}$, it follows that Algorithm LP_Cocomparability correctly computes and sets (in lines 23-24) $\ell\left(u_{z}, G\left(u_{p}, j, j\right)\right)=\ell\left(u_{z}, G_{u_{z}}^{\left|L_{j}\right|}\left(u_{p}, j, j\right)\right)$ and $P\left(u_{z}, G\left(u_{p}, j, j\right)\right)=P\left(u_{z}, G_{u_{z}}^{\left|L_{j}\right|}\left(u_{p}, j, j\right)\right)$, 
for every vertex $u_{z} \in L_{j}$. Thus, the lemma holds for every subgraph $G\left(u_{p}, i, j\right)$ of $G$ such that $1 \leq i=j \leq k$.

Case B. Consider now the case where $1 \leq i<j \leq k$. To prove that the lemma holds in this case, we use the following claim.

Claim 1 For every induced subgraph $G_{u_{z}}^{r}\left(u_{p}, i, j\right)$ of $G, 1 \leq i<j \leq k$ and $0 \leq r \leq\left|L_{j}\right|$, and for every vertex $u_{y} \in V\left(G_{u_{z}}^{r}\left(u_{p}, i, j\right)\right)$ such that $u_{y} \notin L_{j} \backslash\left\{u_{t}\right\}$, where $u_{t}$ is the last vertex of $L_{j}^{r}\left(u_{z}\right)$, Algorithm LP_Cocomparability correctly computes $\ell\left(u_{y} ; G_{u_{z}}^{r}\left(u_{p}, i, j\right)\right)$ and $P\left(u_{y} ; G_{u_{z}}^{r}\left(u_{p}, i, j\right)\right)$.

Recall that $G\left(u_{p}, i, j\right)=G_{u_{z}}^{\left|L_{j}\right|}\left(u_{p}, i, j\right)$ for any vertex $u_{z} \in L_{j}$. Then, on the one hand, for the length of a longest normal antipath of $G\left(u_{p}, i, j\right)$ with right endpoint a vertex $u_{y} \in V\left(G\left(u_{p}, i, j\right)\right)$ such that $u_{y} \notin L_{j}$, we have that $\mathcal{L}\left(u_{y} ; G\left(u_{p}, i, j\right)\right)=\mathcal{L}\left(u_{y} ; G_{u_{z}}^{\left|L_{j}\right|}\left(u_{p}, i, j\right)\right)$, where $u_{z}$ is any vertex of $L_{j}$. Thus, from Claim 1 we obtain that $\ell\left(u_{y} ; G_{u_{z}}^{\left|L_{j}\right|}\left(u_{p}, i, j\right)\right)=\mathcal{L}\left(u_{y} ; G\left(u_{p}, i, j\right)\right)$, where $u_{z}$ is any vertex of $L_{j}$. It is easy to see that, for every vertex $u_{y}$ of $G\left(u_{p}, i, j\right)$ such that $u_{y} \notin L_{j}$, Algorithm LP_Cocomparability sets (in lines 26-28) $\ell\left(u_{y} ; G\left(u_{p}, i, j\right)\right)=\ell\left(u_{y} ; G_{u_{z}}^{\left|L_{j}\right|}\left(u_{p}, i, j\right)\right)$ and $P\left(u_{y} ; G\left(u_{p}, i, j\right)\right)=P\left(u_{y} ; G_{u_{z}}^{\left|L_{j}\right|}\left(u_{p}, i, j\right)\right)$, where $u_{z}$ is any vertex of $L_{j}$.

On the other hand, for the length of a longest normal antipath of $G\left(u_{p}, i, j\right)$ with right endpoint a vertex $u_{z} \in L_{j}$, from Claim 1 we obtain that $\ell\left(u_{z} ; G_{u_{z}}^{\left|L_{j}\right|}\left(u_{p}, i, j\right)\right)=\mathcal{L}\left(u_{z} ; G\left(u_{p}, i, j\right)\right)$. Since the procedure process () is executed for every vertex $u_{z} \in L_{j}$, i.e., for every subgraph $\left.G_{u_{z}}^{r}\left(u_{p}, i, j\right)\right)$, it follows that Algorithm LP_Cocomparability correctly computes and sets (in lines 23-24) $\ell\left(u_{z} ; G\left(u_{p}, i, j\right)\right)=\ell\left(u_{z} ; G_{u_{z}}^{\left|L_{j}\right|}\left(u_{p}, i, j\right)\right)$ and $P\left(u_{z} ; G\left(u_{p}, i, j\right)\right)=P\left(u_{z} ; G_{u_{z}}^{\left|L_{j}\right|}\left(u_{p}, i, j\right)\right)$ for every vertex $u_{z} \in L_{j}$.

It is now clear that Algorithm LP_Cocomparability correctly computes the length of a longest normal antipath of $G\left(u_{p}, i, j\right)$ with right endpoint a vertex $u_{y}$, for every vertex $u_{y} \in V\left(G\left(u_{p}, i, j\right)\right)$. This proves the lemma.

We next prove by induction that the process () of algorithm LP_Cocomparability, i.e., procedures bridge() and append(), correctly computes the length $\ell\left(u_{y} ; G_{u_{z}}^{r}\left(u_{p}, i, j\right)\right)$ of a longest normal antipath of $G_{u_{z}}^{r}\left(u_{p}, i, j\right)$ with right endpoint the vertex $u_{y}$, and the corresponding antipath $P\left(u_{y} ; G_{u_{z}}^{r}\left(u_{p}, i, j\right)\right)$.

\section{Proof of Claim 1.}

For proving the claim we use the induction hypothesis of Lemma 4.3. That is, we assume that for every induced subgraph $G\left(u_{p}, i^{\prime}, j^{\prime}\right)$ of $G, 1 \leq i^{\prime} \leq j^{\prime} \leq j-1 \leq k-1$, and for every vertex $u_{y} \in V\left(G\left(u_{p}, i^{\prime}, j^{\prime}\right)\right)$, the value $\ell\left(u_{y} ; G\left(u_{p}, i^{\prime}, j^{\prime}\right)\right)$ computed by Algorithm LP_Cocomparability is equal to the length $\mathcal{L}\left(u_{y} ; G\left(u_{p}, i^{\prime}, j^{\prime}\right)\right)$ of a longest normal antipath of $G\left(u_{p}, i^{\prime}, j^{\prime}\right)$ with right endpoint the vertex $u_{y}$ and, also, the corresponding computed antipath $P\left(u_{y} ; G\left(u_{p}, i^{\prime}, j^{\prime}\right)\right)$ is a longest normal antipath of $G\left(u_{p}, i^{\prime}, j^{\prime}\right)$ with right endpoint the vertex $u_{y}$.

Let $G_{u_{z}}^{r}\left(u_{p}, i, j\right)$ be an induced subgraph of $G$ such that $1 \leq i<j \leq k$ and $0 \leq r \leq\left|L_{j}\right|$. We prove the claim by induction on the index $r, 0 \leq r \leq\left|L_{j}\right|$, i.e., we prove that for every induced subgraph $G_{u_{z}}^{r}\left(u_{p}, i, j\right)$ of $G, 1 \leq i<j \leq k$ and $0 \leq r \leq\left|L_{j}\right|$, and for every vertex $u_{y} \in V\left(G_{u_{z}}^{r}\left(u_{p}, i, j\right)\right)$ such that $u_{y} \notin L_{j} \backslash\left\{u_{t}\right\}$, where $u_{t}$ is the last vertex of $L_{j}^{r}\left(u_{z}\right)$, Algorithm LP_Cocomparability correctly computes $\ell\left(u_{y} ; G_{u_{z}}^{r}\left(u_{p}, i, j\right)\right)$ and $P\left(u_{y} ; G_{u_{z}}^{r}\left(u_{p}, i, j\right)\right)$.

To this end, we distinguish three cases concerning the position of the vertex $u_{y}$ in the ordering $\sigma$. In each case, we examine first the optimum value of the length $\mathcal{L}\left(u_{y} ; G_{u_{z}}^{r}\left(u_{p}, i, j\right)\right)$ of a longest normal antipath of $G_{u_{z}}^{r}\left(u_{p}, i, j\right)$ with right endpoint the vertex $u_{y}$, and then we compare this value to the value $\ell\left(u_{y} ; G_{u_{z}}^{r}\left(u_{p}, i, j\right)\right)$ computed by Algorithm LP_Cocomparability; we also compare the corresponding antipaths.

Induction basis. Consider first the case where $r=0$, i.e., $L_{j}^{0}=\emptyset$. Since in this claim we examine the case where $i \neq j$, from Definition 3.2 we obtain that $G_{u_{z}}^{0}\left(u_{p}, i, j\right)=G\left(u_{p}, i, j-1\right)$. Therefore, 
it is easy to see that for every subgraph $G_{u_{z}}^{0}\left(u_{p}, i, j\right)$, and for every vertex $u_{y} \in V\left(G_{u_{z}}^{0}\left(u_{p}, i, j\right)\right)$, the length $\mathcal{L}\left(u_{y} ; G_{u_{z}}^{0}\left(u_{p}, i, j\right)\right)$ is equal to $\mathcal{L}\left(u_{y} ; G\left(u_{p}, i, j-1\right)\right)$.

Algorithm LP_Cocomparability sets (in lines 8-11) $\ell\left(u_{y} ; G_{u_{z}}^{0}\left(u_{p}, i, j\right)\right)=\ell\left(u_{y} ; G\left(u_{p}, i, j-1\right)\right)$ and $P\left(u_{y} ; G_{u_{z}}^{0}\left(u_{p}, i, j\right)\right)=P\left(u_{y} ; G\left(u_{p}, i, j-1\right)\right)$, for every vertex $u_{y} \in V\left(G_{u_{z}}^{0}\left(u_{p}, i, j\right)\right)$. Since by the induction hypothesis of Lemma 4.3, Algorithm LP_Cocomparability correctly computes the values of $\ell\left(u_{y} ; G\left(u_{p}, i, j-1\right)\right)$ and $P\left(u_{y} ; G\left(u_{p}, i, j-1\right)\right)$, it follows that the algorithm also correctly computes the values of $\ell\left(u_{y} ; G_{u_{z}}^{0}\left(u_{p}, i, j\right)\right)$ and $P\left(u_{y} ; G_{u_{z}}^{0}\left(u_{p}, i, j\right)\right)$. Therefore, the claim holds for $r=0$.

Induction hypothesis. Suppose now that the claim holds for every index $\ell, 0 \leq \ell \leq r-1 \leq$ $\left|L_{j}\right|-1$.

Induction step. We will now prove that the claim holds for the subgraph $G_{u_{z}}^{r}\left(u_{p}, i, j\right)$ of $G$, $1 \leq r \leq\left|L_{j}\right|$. To this end, we distinguish three cases concerning the position of the vertex $u_{y}$ in the ordering $\sigma$ :

Case 1: $u_{y} \in H_{\ell}, i \leq \ell \leq f\left(u_{t}\right)$,

Case 2: $u_{y} \in H_{\ell}, f\left(u_{t}\right)+1 \leq \ell \leq j-1$, and

Case 3: $u_{y}=u_{t}$.

Note that Cases 1 and 2 correspond to the procedure bridge() of algorithm LP_Cocomparability, while Case 3 corresponds to procedure append().

Case 1. We consider first the case where $u_{y} \in H_{\ell}$ and $i \leq \ell \leq f\left(u_{t}\right)$. In this case we have $\mathcal{L}\left(u_{y} ; G_{u_{z}}^{r}\left(u_{p}, i, j\right)\right)=\mathcal{L}\left(u_{y} ; G_{u_{z}}^{r-1}\left(u_{p}, i, j\right)\right)$, since from Definition 2.2 and Notation 3.1 we obtain that $u_{t}$ does not belong to any normal antipath with right endpoint a vertex $u_{y} \in H_{\ell}, i \leq \ell \leq f\left(u_{t}\right)$.

In this case, Algorithm LP_Cocomparability computes and sets (in lines 8-14) $\ell\left(u_{y} ; G_{u_{z}}^{r}\left(u_{p}, i, j\right)\right)=\ell\left(u_{y} ; G_{u_{z}}^{r-1}\left(u_{p}, i, j\right)\right)$ for the length of a longest normal antipath of $G_{u_{z}}^{r}\left(u_{p}, i, j\right)$ with right endpoint a vertex $u_{y} \in H_{\ell}, i \leq \ell \leq f\left(u_{t}\right)$; the algorithm also computes the corresponding antipath. Note that, this computation is done during the initialization (in lines 8-14), and these values do not change during the process() of the algorithm, since $u_{y} \in H_{\ell}$ and $\ell<f\left(u_{t}\right)+1$. Since by the induction hypothesis Algorithm LP_Cocomparability correctly computes and sets $\ell\left(u_{y} ; G_{u_{z}}^{r-1}\left(u_{p}, i, j\right)=\mathcal{L}\left(u_{y} ; G_{u_{z}}^{r-1}\left(u_{p}, i, j\right)\right.\right.$, for every vertex $u_{y} \in G_{u_{z}}^{r-1}\left(u_{p}, i, j\right)$ such that $u_{y} \notin L_{j}$, it follows that Algorithm LP_Cocomparability correctly computes the values of $\ell\left(u_{y} ; G_{u_{z}}^{r}\left(u_{p}, i, j\right)\right)$ and $P\left(u_{y} ; G_{u_{z}}^{r}\left(u_{p}, i, j\right)\right)$.

Case 2. We consider next the case where $u_{y} \in H_{h}$ and $f\left(u_{t}\right)+1 \leq h \leq j-1$. Let $P=\left(u_{x^{\prime}}, \ldots, u_{y}\right)$ be a longest normal antipath of $G_{u_{z}}^{r}\left(u_{p}, i, j\right)$ which has $u_{y}$ as its right endpoint. We now distinguish two cases: (I) the case where $P$ contains the vertex $u_{t}$, and (II) the case where $P$ does not contain the vertex $u_{t}$.

2(I) Consider first the case where $P$ contains the vertex $u_{t}$. Assume first that $P=\left(u_{t}, u_{y}\right)$ is a longest normal antipath of $G_{u_{z}}^{r}\left(u_{p}, i, j\right)$ with right endpoint the vertex $u_{y}$. From Definition 2.2 we obtain that $P$ is not normal; this is a contradiction to our assumption on $P$.

Assume now that $P=\left(u_{x^{\prime}}, \ldots, u_{x}, u_{t}, u_{y^{\prime}}, \ldots, u_{y}\right)=\left(P_{1}, u_{t}, P_{2}\right)$ is a longest normal antipath of $G_{u_{z}}^{r}\left(u_{p}, i, j\right)$ with right endpoint the vertex $u_{y}$. From Lemma 4.1 , we obtain that $P_{1}=\left(u_{x^{\prime}}, \ldots, u_{x}\right)$ and $P_{2}=\left(u_{y^{\prime}}, \ldots, u_{y}\right)$ are normal antipaths of $G_{u_{z}}^{r}\left(u_{p}, i, j\right)$ and, in fact, of $G_{u_{z}}^{r-1}\left(u_{p}, i, j\right)$. We next prove the following results (Claims 2,3 , and 4 ) for the antipaths $P, P_{1}$, and $P_{2}$.

Claim 2 Let $P, P_{1}$, and $P_{2}$ be the antipaths of Case 2(I). Then, for every vertex $u_{s} \in V\left(P_{2}\right)$ we have $u_{x}<_{\sigma} u_{s}$ and $u_{x} u_{s} \in E(G)$, where $u_{x}$ is the right endpoint of $P_{1}$.

Proof of Claim 2. Let $P=\left(P_{1}, u_{t}, P_{2}\right)$ and let $u_{x}$ be the right endpoint of $P_{1}$. Since $u_{t} \in L_{j}$ and $P$ is an antipath of $G_{u_{z}}^{r}\left(u_{p}, i, j\right)$, it follows that $u_{s} \leq_{\sigma} u_{t}$ for every vertex $u_{s} \in V\left(P_{2}\right)$. 
(i) Consider first the case where $u_{s}$ is a vertex of $P_{2}$ such that $u_{s}<_{\sigma} u_{t}$. Since $P$ is normal and $u_{t}$ is the next vertex of $u_{x}$ in $P$, it follows that $u_{x} u_{s} \in E(G)$ for every vertex $u_{s} \in V\left(P_{2}\right)$ such that $u_{s}<_{\sigma} u_{t}$. Since $P$ is normal, $u_{x} u_{s} \in E(G)$, and $u_{x}$ appears before $u_{s}$ in $P$, from Lemma 2.4 we obtain that $u_{x}<_{\sigma} u_{s}$, for every vertex $u_{s} \in V\left(P_{2}\right)$ such that $u_{s}<_{\sigma} u_{t}$. Therefore, we have proved that for every vertex $u_{s} \in V\left(P_{2}\right)$ such that $u_{s}<_{\sigma} u_{t}$, we have $u_{x}<_{\sigma} u_{s}$ and $u_{x} u_{s} \in E(G)$.

(ii) Consider now the case where $u_{s}$ is a vertex of $P_{2}$ such that $u_{s}={ }_{\sigma} u_{t}$. Since $u_{y}$ is a vertex of $P_{2}$ such that $u_{y}<_{\sigma} u_{t}$, from case (i) of Claim 2 we obtain that $u_{x}<_{\sigma} u_{y}$. Since $u_{x}<_{\sigma} u_{y}<_{\sigma} u_{t}={ }_{\sigma} u_{s}$, it follows that $u_{x}<_{\sigma} u_{s}$, for every vertex $u_{s} \in V\left(P_{2}\right)$ such that $u_{s}={ }_{\sigma} u_{t}$. It is left to show that the property $u_{x} u_{s} \in E(G)$ holds for every vertex $u_{s} \in V\left(P_{2}\right)$ such that $u_{s}={ }_{\sigma} u_{t}$. Assume that $P$ is an antipath for which this property does not hold. We next show that there exists a longest normal antipath $P^{\prime}$ of $G_{u_{z}}^{r}\left(u_{p}, i, j\right)$ with right endpoint the vertex $u_{y}$, such that $P^{\prime}=\left(P_{1}, P_{2}^{\prime}\right)$ and $V\left(P^{\prime}\right)=V(P)$, for which the property $u_{x} u_{s} \in E(G)$ holds for every vertex $u_{s} \in V\left(P_{2}\right)$ such that $u_{s}={ }_{\sigma} u_{t}$.

Let $u_{s}$ be a vertex of $P_{2}$ such that $u_{s}={ }_{\sigma} u_{t}$ and $u_{x} u_{s} \notin E(G)$. Let $P=\left(P_{1}, u_{t}, P_{2}\right)=\left(P_{1}, u_{t}, u_{y^{\prime}} \ldots, u_{s^{\prime}}, u_{s}, u_{s^{\prime \prime}}, \ldots, u_{y}\right)$, and let $u_{s}$ be the last such vertex in $P$. Then $P^{\prime}=\left(P_{1}, P_{2}^{\prime}\right)=\left(P_{1}, u_{s}, u_{y^{\prime}} \ldots, u_{s^{\prime}}, u_{t}, u_{s^{\prime \prime}}, \ldots, u_{y}\right)$ is an antipath, since we next prove that both $u_{t}$ and $u_{s}$ are connected with an antiedge to every vertex of $P_{2}$. To this end, let $u_{q}$ be a vertex of $P_{2}$ such that $q \neq s$. If $u_{q}={ }_{\sigma} u_{t}$, then indeed $u_{q} u_{t} \notin E(G)$ and $u_{q} u_{s} \notin E(G)$. If $u_{q}<_{\sigma} u_{t}$, then from case (i) of Claim 2 we obtain that $u_{x}<_{\sigma} u_{q}$ and $u_{x} u_{q} \in E(G)$. Since $u_{x}<_{\sigma} u_{q}<_{\sigma} u_{t}$, $u_{x} u_{q} \in E(G)$, and $u_{x} u_{t} \notin E(G)$, from the transitivity property we obtain $u_{q} u_{t} \notin E(G)$; using the same arguments we obtain that $u_{q} u_{s} \notin E(G)$. Therefore, since $u_{y^{\prime}}, u_{s^{\prime}}$, and $u_{s^{\prime \prime}}$ are vertices of $P_{2}$, we obtain that $P^{\prime}=\left(P_{1}, u_{s}, u_{y^{\prime}} \ldots, u_{s^{\prime}}, u_{t}, u_{s^{\prime \prime}}, \ldots, u_{y}\right)$ is a longest antipath of $G_{u_{z}}^{r}\left(u_{p}, i, j\right)$ with right endpoint the vertex $u_{y}$. It is easy to see that $P^{\prime}$ is normal, since $P$ is normal and $u_{t}={ }_{\sigma} u_{s}$. Additionally, since $P^{\prime}$ is normal, it is easy to obtain that the property of case (i) of Claim 2 holds for $P^{\prime}$ as well, where $P^{\prime}=\left(P_{1}, u_{s}, \widetilde{P_{2}}\right)$. By repeating the above procedure we can obtain a longest normal antipath $P^{\prime}=\left(P_{1}^{\prime}, u_{t}, P_{2}^{\prime}\right)$ with right endpoint the vertex $u_{y}$ such that $u_{x} u_{s} \in E(G)$ for every vertex $u_{s} \in V\left(P_{2}^{\prime}\right)$ such that $u_{s}={ }_{\sigma} u_{t}$, where $u_{x}$ is the last vertex of $P_{1}^{\prime}$.

Therefore, we may assume without loss of generality that $P=\left(u_{x^{\prime}}, \ldots, u_{x}, u_{t}, u_{y^{\prime}}, \ldots, u_{y}\right)=\left(P_{1}, u_{t}, P_{2}\right)$ is a longest normal antipath of $G_{u_{z}}^{r}\left(u_{p}, i, j\right)$ with right endpoint the vertex $u_{y}$, with the property that $u_{x} u_{s} \in E(G)$ for every vertex $u_{s} \in V\left(P_{2}\right)$ such that $u_{s}={ }_{\sigma} u_{t}$. Thus, we have proved that $u_{x}<_{\sigma} u_{s}$ and $u_{x} u_{s} \in E(G)$, for every vertex $u_{s} \in V\left(P_{2}\right) ; \quad Q E D$ of Claim 2 .

Claim 3 Let $P, P_{1}$, and $P_{2}$ be the antipaths of Case 2(I). Then, $P_{1}$ is a normal antipath of $G_{u_{z}}^{r-1}\left(u_{p}, i, j\right)$ which has $u_{x}$ as its right endpoint, and $P_{2}$ is a normal antipath of $G_{u_{z}}^{r-1}\left(u_{x}, \ell+1, j\right)$ which has $u_{y}$ as its right endpoint.

Proof of Claim 3. By assumption, for every vertex $u_{q} \in V\left(P_{1}\right)$ we have $u_{q} \in V\left(G_{u_{z}}^{r-1}\left(u_{p}, i, j\right)\right)$, and $u_{x}$ is the right endpoint of $P_{1}$.

Since $u_{x}<_{\sigma} u_{y}$ and by assumption $u_{y} \in H_{h}, f\left(u_{t}\right)+1 \leq h \leq j-1$, we obtain that $u_{x} \in H_{\ell}$, where $f\left(u_{t}\right) \leq \ell \leq j-2$. Then, from Claim 2 we obtain that $u_{s} \in H_{h}, \ell+1 \leq h \leq j$, for every vertex $u_{s} \in V\left(P_{2}\right)$. Additionally, since from Claim 2 we have $u_{x} u_{s} \in E(G)$ for every vertex $u_{s} \in V\left(P_{2}\right)$, it follows from Definition 3.2 that $u_{s} \in V\left(G_{u_{z}}^{r-1}\left(u_{x}, \ell+1, j\right)\right)$ for every vertex $u_{s} \in V\left(P_{2}\right)$. Note that, every vertex $u_{s}$ of $G_{u_{z}}^{r-1}\left(u_{x}, \ell+1, j\right)$ is also a vertex of $G_{u_{z}}^{r-1}\left(u_{p}, i, j\right)$. Indeed, since $i<\ell+1 \leq j$, and since $u_{p}<_{\sigma} u_{x}<_{\sigma} u_{s}, u_{p} u_{x} \in E(G)$, and $u_{x} u_{s} \in E(G)$, from the transitivity property we obtain that $u_{p} u_{s} \in E(G)$. Therefore, we have shown that every vertex of $P_{2}$ belongs to $G_{u_{z}}^{r-1}\left(u_{x}, \ell+1, j\right)$; QED of Claim 3.

Claim 4 If $P_{1}$ is a normal antipath of $G_{u_{z}}^{r-1}\left(u_{p}, i, j\right)$ which has $u_{x}$ as its right endpoint, and $P_{2}$ is a normal antipath of $G_{u_{z}}^{r-1}\left(u_{x}, \ell+1, j\right)$ which has $u_{y}$ as its right endpoint, then $V\left(P_{1}\right) \cap V\left(P_{2}\right)=\emptyset$. 
Proof of Claim 4. Let $\mathcal{H}_{2}$ be the subgraph of $G_{u_{z}}^{r}\left(u_{p}, i, j\right)$ induced by $V\left(\mathcal{H}_{2}\right)=V\left(G_{u_{z}}^{r-1}\left(u_{x}, \ell+1, j\right)\right)$; recall from Claim 3 that every vertex of $P_{2}$ belongs to $\mathcal{H}_{2}$.

Let $u_{q}$ be a vertex of $P_{1}$. If $u_{q} \leq_{\sigma} u_{x}$, then $u_{q} \in H_{d}$ and $d \leq \ell$; thus, from Definition 3.2 we obtain that $u_{q} \notin V\left(\mathcal{H}_{2}\right)=V\left(G_{u_{z}}^{r-1}\left(u_{x}, \ell+1, j\right)\right)$. Consider now the case where $u_{q}$ is a vertex of $P_{1}$ such that $u_{x}<_{\sigma} u_{q}$. Since $P_{1}$ is a normal antipath, $u_{x}<_{\sigma} u_{q}$, and $u_{q}$ appears before $u_{x}$ in $P_{1}$, from Lemma 2.4 we obtain that $u_{x} u_{q} \notin E(G)$. Therefore, from Definition 3.2 we obtain again that $u_{q} \notin$ $V\left(\mathcal{H}_{2}\right)=V\left(G_{u_{z}}^{r-1}\left(u_{x}, \ell+1, j\right)\right)$. Therefore, we have proved that no vertex of $P_{1}$ belongs to $\mathcal{H}_{2}$. Let $\mathcal{H}_{1}$ be the subgraph of $G_{u_{z}}^{r}\left(u_{p}, i, j\right)$ induced by $V\left(\mathcal{H}_{1}\right)=V\left(G_{u_{z}}^{r-1}\left(u_{p}, i, j\right)\right) \backslash V\left(G_{u_{z}}^{r-1}\left(u_{x}, \ell+1, j\right)\right)$. Thus, we have shown that every vertex of $P_{1}$ belongs to $\mathcal{H}_{1}$.

Therefore, we have shown that $V\left(P_{1}\right) \subseteq V\left(\mathcal{H}_{1}\right), V\left(P_{2}\right) \subseteq V\left(\mathcal{H}_{2}\right)$, and $V\left(\mathcal{H}_{1}\right) \cap V\left(\mathcal{H}_{2}\right)=\emptyset$. It is easy to see that $V\left(P_{1}\right) \cap V\left(P_{2}\right)=\emptyset ; \quad$ QED of Claim 4.

Since $P=\left(P_{1}, u_{t}, P_{2}\right)$ is a longest normal antipath of $G_{u_{z}}^{r}\left(u_{p}, i, j\right)$ with right endpoint the vertex $u_{y}$, and since the antipaths $P_{1}$ and $P_{2}$ belong to two disjoint induced subgraphs of $G_{u_{z}}^{r}\left(u_{p}, i, j\right)$, it follows that $P_{1}$ is a longest normal antipath of $H_{1}$ with right endpoint the vertex $u_{x}$, and that $P_{2}$ is a longest normal antipath of $H_{2}$ with right endpoint the vertex $u_{y}$. Note that, $u_{t}$ is connected with an antiedge to every vertex $u_{s}$ of $H_{2}$ and, thus, also of $P_{2}$. Indeed, in the case where $u_{s}={ }_{\sigma} u_{t}$ this is straightforward. In the case where $u_{s}<_{\sigma} u_{t}$, then from Claim 2 we have and $u_{x}<_{\sigma} u_{s}<_{\sigma} u_{t}$ and $u_{x} u_{s} \in E(G)$ for every vertex $u_{s}$ of $P_{2}$; since $u_{x} u_{t} \notin E(G)$, from the transitivity property we obtain that $u_{s} u_{t} \notin E(G)$ for every vertex $u_{s}$ of $P_{2}$.

Therefore, since $H_{2}=G_{u_{z}}^{r-1}\left(u_{x}, \ell+1, j\right)$, we obtain that $\left|P_{2}\right|=\mathcal{L}\left(u_{y} ; G_{u_{z}}^{r-1}\left(u_{x}, \ell+1, j\right)\right)$. We will now show that $\left|P_{1}\right|=\mathcal{L}\left(u_{x} ; G_{u_{z}}^{r-1}\left(u_{p}, i, j\right)\right)$. To this end, let $P_{0}$ be a longest normal antipath of $G_{u_{z}}^{r-1}\left(u_{p}, i, j\right)$ with right endpoint the vertex $u_{x}$. Assume that there exists a vertex $u_{s} \in V\left(P_{0}\right)$ such that $u_{s} \in V\left(\mathcal{H}_{2}\right)=V\left(G_{u_{z}}^{r-1}\left(u_{x}, \ell+1, j\right)\right)$. Since $u_{x} \in H_{\ell}$, it follows that $u_{x}<_{\sigma} u_{s}$ and $u_{x} u_{s} \in E(G)$. Since $P_{0}$ is normal, from Lemma 2.4 we obtain that $u_{x}$ appears before $u_{s}$ in $P_{0}$. This comes to a contradiction to our assumption that $u_{x}$ is the right endpoint of $P_{0}$. Thus, no vertex of $P_{0}$ belongs to $\mathcal{H}_{2}$. Thus, $V\left(P_{0}\right) \subseteq V\left(\mathcal{H}_{1}\right)$, and since $P_{1}$ is a longest normal antipath of $H_{1}$ with right endpoint the vertex $u_{x}$, we obtain that $\left|P_{0}\right| \subseteq\left|P_{1}\right|$. Additionally, since $\mathcal{H}_{1}$ is an induced subgraph of $G_{u_{z}}^{r-1}\left(u_{p}, i, j\right)$, we obtain that $\left|P_{1}\right| \subseteq\left|P_{0}\right|$. Thus, $\left|P_{0}\right|=\left|P_{1}\right|$ and, therefore, $P_{1}$ is a longest normal antipath of $G_{u_{z}}^{r-1}\left(u_{p}, i, j\right)$ with right endpoint the vertex $u_{x}$. Thus, $\left|P_{1}\right|=\mathcal{L}\left(u_{x} ; G_{u_{z}}^{r-1}\left(u_{p}, i, j\right)\right)$.

Therefore, if $P=\left(P_{1}, u_{t}, P_{2}\right)$ is a longest normal antipath of $G_{u_{z}}^{r}\left(u_{p}, i, j\right)$ with right endpoint a vertex $u_{y} \in H_{h}, f\left(u_{t}\right)+1 \leq h \leq j-1$, we have shown that $|P|=\mathcal{L}\left(u_{y} ; G_{u_{z}}^{r}\left(u_{p}, i, j\right)\right)=\mathcal{L}\left(u_{x} ; G_{u_{z}}^{r-1}\left(u_{p}, i, j\right)\right)+\mathcal{L}\left(u_{y} ; G_{u_{z}}^{r-1}\left(u_{x}, \ell+1, j\right)\right)+1$ and $P=$ $\mathcal{P}\left(u_{y} ; G_{u_{z}}^{r}\left(u_{p}, i, j\right)\right)=\left(\mathcal{P}\left(u_{x} ; G_{u_{z}}^{r-1}\left(u_{p}, i, j\right)\right), u_{t}, \mathcal{P}\left(u_{y} ; G_{u_{z}}^{r-1}\left(u_{x}, \ell+1, j\right)\right)\right)$.

We next examine the results computed by Algorithm LP_Cocomparability in Case 2(I). Let $P\left(u_{y} ; G_{u_{z}}^{r}\left(u_{p}, i, j\right)\right)$ be the antipath of $G_{u_{z}}^{r}\left(u_{p}, i, j\right)$ with right endpoint a vertex $u_{y}$ computed by Algorithm LP_Cocomparability, in the case where $u_{y} \in H_{h}, f\left(u_{t}\right)+1 \leq h \leq j-1$. Note that the antipath $P\left(u_{y} ; G_{u_{z}}^{r}\left(u_{p}, i, j\right)\right)$, which is computed by the algorithm with the procedure bridge(), contains the vertex $u_{t}$. In fact, Algorithm LP_Cocomparability computes and sets $P\left(u_{y} ; G_{u_{z}}^{r}\left(u_{p}, i, j\right)\right)=\left(P_{1}^{\prime}, u_{t}, P_{2}^{\prime}\right)$, where $u_{t}$ is the last vertex of $L_{j}^{r}\left(u_{z}\right)$, and for the two antipaths $P_{1}^{\prime}$ and $P_{2}^{\prime}$ we have: $P_{1}^{\prime}=P\left(u_{x} ; G_{u_{z}}^{r-1}\left(u_{p}, i, j\right)\right)$, where $u_{x} \in H_{\ell}, f\left(u_{t}\right) \leq \ell \leq j-2$, and $u_{x} u_{t} \notin E(G)$, and $P_{2}^{\prime}=P\left(u_{y} ; G_{u_{z}}^{r-1}\left(u_{x}, \ell+1, j\right)\right)$, where $u_{y} \in H_{h}, \ell+1 \leq h \leq j-1$. Actually, in this case, Algorithm LP_Cocomparability computes with the procedure bridge() the value $w_{1}+w_{2}+1=\left|P_{1}^{\prime}\right|+\left|P_{2}^{\prime}\right|+1$, for every vertex $u_{x}$ such that $u_{x} \in H_{\ell}, f\left(u_{t}\right) \leq \ell \leq j-2$, and $u_{x} u_{t} \notin E(G)$, and sets $\ell\left(u_{y} ; G_{u_{z}}^{r}\left(u_{p}, i, j\right)\right)$ to be equal to the maximum among these values. Also, Algorithm LP_on_H computes the corresponding antipath $P\left(u_{y} ; G_{u_{z}}^{r}\left(u_{p}, i, j\right)\right)=\left(P_{1}^{\prime}, u_{t}, P_{2}^{\prime}\right)$.

By the induction hypothesis, we obtain that Algorithm LP_Cocomparability has correctly computed the values $P_{1}^{\prime}=P\left(u_{x} ; G_{u_{z}}^{r-1}\left(u_{p}, i, j\right)\right)$ and $P_{2}^{\prime}=P\left(u_{y} ; G_{u_{z}}^{r-1}\left(u_{x}, \ell+1, j\right)\right)$, i.e., $P_{1}^{\prime}=$ $\mathcal{P}\left(u_{x} ; G_{u_{z}}^{r-1}\left(u_{p}, i, j\right)\right)$ and $P_{2}^{\prime}=\mathcal{P}\left(u_{y} ; G_{u_{z}}^{r-1}\left(u_{x}, \ell+1, j\right)\right)$. Therefore, from Claim 3 we obtain that $V\left(P_{1}^{\prime}\right) \cap V\left(P_{2}^{\prime}\right)=\emptyset$. Then, from Lemma 4.2 we obtain that the antipath $P\left(u_{y} ; G_{u_{z}}^{r}\left(u_{p}, i, j\right)\right)=$ 
$\left(P_{1}^{\prime}, u_{t}, P_{2}^{\prime}\right)$ computed by Algorithm LP_Cocomparability is a normal antipath of $G_{u_{z}}^{r}\left(u_{p}, i, j\right)$ with right endpoint the vertex $u_{y}$. Moreover, since Algorithm LP_Cocomparability computes with the procedure bridge () the value $\ell\left(u_{x} ; G_{u_{z}}^{r-1}\left(u_{p}, i, j\right)\right)+\ell\left(u_{y} ; G_{u_{z}}^{r-1}\left(u_{x}, \ell+1, j\right)\right)+1$, for every vertex $u_{x}$ such that $u_{x} \in H_{\ell}, f\left(u_{t}\right) \leq \ell \leq j-2$, and $u_{x} u_{t} \notin E(G)$, and sets $\ell\left(u_{y} ; G_{u_{z}}^{r}\left(u_{p}, i, j\right)\right)$ to be equal to the maximum among these values, it follows that $\ell\left(u_{y} ; G_{u_{z}}^{r}\left(u_{p}, i, j\right)\right)=\mathcal{L}\left(u_{y} ; G_{u_{z}}^{r}\left(u_{p}, i, j\right)\right)$. Also, the corresponding antipath $P\left(u_{y} ; G_{u_{z}}^{r}\left(u_{p}, i, j\right)=\left(P\left(u_{x} ; G_{u_{z}}^{r-1}\left(u_{p}, i, j\right)\right), u_{t}, P\left(u_{y} ; G_{u_{z}}^{r-1}\left(u_{x}, \ell+\right.\right.\right.\right.$ $1, j))$ ) computed by Algorithm LP_Cocomparability is a longest normal antipath of $G_{u_{z}}^{r}\left(u_{p}, i, j\right)$ with right endpoint the vertex $u_{y}$.

2(II) Consider now the case where there exists a longest normal antipath $P$ of $G_{u_{z}}^{r}\left(u_{p}, i, j\right)$ with right endpoint the vertex $u_{y}$ which does not contain the vertex $u_{t}$. Then $V(P) \subseteq V\left(G_{u_{z}}^{r-1}\left(u_{p}, i, j\right)\right)$ and, thus, $P$ is a longest normal antipath of $G_{u_{z}}^{r-1}\left(u_{p}, i, j\right)$ with right endpoint the vertex $u_{y}$, i.e., $P=\mathcal{P}\left(u_{y} ; G_{u_{z}}^{r-1}\left(u_{p}, i, j\right)\right)$.

We next examine the results computed by Algorithm LP_Cocomparability in Case 2(II). By the induction hypothesis, we obtain that Algorithm LP_Cocomparability correctly computes $\ell\left(u_{y} ; G_{u_{z}}^{r-1}\left(u_{p}, i, j\right)\right)=\mathcal{L}\left(u_{y} ; G_{u_{z}}^{r-1}\left(u_{p}, i, j\right)\right)$ for every vertex $u_{y} \in G_{u_{z}}^{r-1}\left(u_{p}, i, j\right)$ such that $u_{y} \notin L_{j} \backslash\left\{u_{t}\right\}$. Observe first that during the initialization (in lines 8-14) the algorithm sets $\ell\left(u_{y} ; G_{u_{z}}^{r}\left(u_{p}, i, j\right)\right)=\ell\left(u_{y} ; G_{u_{z}}^{r-1}\left(u_{p}, i, j\right)\right)$ for every vertex $u_{y} \in H_{h}, f\left(u_{t}\right)+1 \leq h \leq j-1$. Next, it suffices to show that these values do not change during the execution of the process () .

From Lemma 4.2 (since from Claim 4 we have $V\left(P_{1}^{\prime}\right) \cap V\left(P_{2}^{\prime}\right)=\emptyset$ ), we obtain that the antipaths $\left(P_{1}^{\prime}, u_{t}, P_{2}^{\prime}\right)$ constructed by Algorithm LP_Cocomparability, during the execution of the procedure bridge (), are normal antipaths of $G_{u_{z}}^{r}\left(u_{p}, i, j\right)$ with right endpoint a vertex $u_{y}$. Therefore, since we have assumed that the longest normal antipath $P$ of $G_{u_{z}}^{r}\left(u_{p}, i, j\right)$ with right endpoint the vertex $u_{y}$ does not contain the vertex $u_{t}$, it directly follows that no (normal) antipath $\left(P_{1}^{\prime}, u_{t}, P_{2}^{\prime}\right)$ with right endpoint the vertex $u_{y}$ which is constructed with the procedure bridge () is longer than $P$. Thus, since $|P|$ is the initial value given to $\ell\left(u_{y} ; G_{u_{z}}^{r}\left(u_{p}, i, j\right)\right)$ (during the initialization in lines 8-14), it follows that the statement $w_{1}+w_{2}+1>\ell\left(u_{y} ; G_{u_{z}}^{r}\left(u_{p}, i, j\right)\right)$ (in the procedure bridge()) is false for every vertex $u_{x} \in H_{\ell}$ such that $f\left(u_{t}\right) \leq \ell \leq h-1$ and $u_{t} u_{x} \notin E(G)$. Therefore, the initial value of $\ell\left(u_{y} ; G_{u_{z}}^{r}\left(u_{p}, i, j\right)\right)$ does not change during the execution of the process () .

Thus, Algorithm LP_Cocomparability correctly computes $\ell\left(u_{y} ; G_{u_{z}}^{r}\left(u_{p}, i, j\right)\right)=$ $\ell\left(u_{y} ; G_{u_{z}}^{r-1}\left(u_{p}, i, j\right)\right)$ and $P\left(u_{y} ; G_{u_{z}}^{r}\left(u_{p}, i, j\right)\right)=P\left(u_{y} ; G_{u_{z}}^{r-1}\left(u_{p}, i, j\right)\right)$; recall that, by the induction hypothesis, $\ell\left(u_{y} ; G_{u_{z}}^{r-1}\left(u_{p}, i, j\right)\right)=\mathcal{L}\left(u_{y} ; G_{u_{z}}^{r-1}\left(u_{p}, i, j\right)\right)$ and $P\left(u_{y} ; G_{u_{z}}^{r-1}\left(u_{p}, i, j\right)\right)=$ $\mathcal{P}\left(u_{y} ; G_{u_{z}}^{r-1}\left(u_{p}, i, j\right)\right)$.

Concluding, in both Cases 2(I) and 2(II), we have proved that the antipath $P\left(u_{y} ; G_{u_{z}}^{r}\left(u_{p}, i, j\right)\right)$ computed by Algorithm LP_Cocomparability is a longest normal antipath $\mathcal{P}\left(u_{y} ; G_{u_{z}}^{r}\left(u_{p}, i, j\right)\right)$ of $G_{u_{z}}^{r}\left(u_{p}, i, j\right)$ with $u_{y}$ as its right endpoint, and $\ell\left(u_{y} ; G_{u_{z}}^{r}\left(u_{p}, i, j\right)\right)=\mathcal{L}\left(u_{y} ; G_{u_{z}}^{r}\left(u_{p}, i, j\right)\right)$. Thus, the claim holds in Case 2.

Case 3. Consider now the case where $u_{y}=u_{t}$.

3(I) Assume first that $u_{t}$ has no antineighbors in $G_{u_{z}}^{r}\left(u_{p}, i, j\right)$. Then $\mathcal{P}\left(u_{t} ; G_{u_{z}}^{r}\left(u_{p}, i, j\right)\right)=\left(u_{t}\right)$ is a longest normal antipath of $G_{u_{z}}^{r}\left(u_{p}, i, j\right)$ with right endpoint the vertex $u_{t}$.

Since we examine the case where $i \neq j$, it is easy to see that Algorithm LP_Cocomparability sets (in lines 19-20) $\ell\left(u_{t} ; G_{u_{z}}^{r}\left(u_{p}, i, j\right)\right)=1$ and $P\left(u_{t} ; G_{u_{z}}^{r}\left(u_{p}, i, j\right)\right)=\left(u_{t}\right)$. Since $u_{t}$ has no antineighbors in $G_{u_{z}}^{r}\left(u_{p}, i, j\right)$, it follows that $r=1$ and $f\left(u_{t}\right)=j$. Thus, the initial value of $\ell\left(u_{t} ; G_{u_{z}}^{r}\left(u_{p}, i, j\right)\right)$ does not change during the execution of the process (). Therefore, Algorithm LP_Cocomparability correctly computes the values of $\ell\left(u_{t} ; G_{u_{z}}^{r}\left(u_{p}, i, j\right)\right)$ and $P\left(u_{t} ; G_{u_{z}}^{r}\left(u_{p}, i, j\right)\right)$ in the case where $u_{t}$ has no antineighbors in $G_{u_{z}}^{r}\left(u_{p}, i, j\right)$.

3(II) Assume now that $u_{t}$ has at least one antineighbor in $G_{u_{z}}^{r}\left(u_{p}, i, j\right)$. Let $P=$ $\left(u_{x^{\prime}}, \ldots, u_{x}, u_{t}\right)=\left(P^{\prime}, u_{t}\right)$ be a longest normal antipath $\mathcal{P}\left(u_{t} ; G_{u_{z}}^{r}\left(u_{p}, i, j\right)\right)$ of $G_{u_{z}}^{r}\left(u_{p}, i, j\right)$ with right endpoint the vertex $u_{t}$. Then, it is easy to see that $P^{\prime}$ is a longest normal antipath of $G_{u_{z}}^{r-1}\left(u_{p}, i, j\right)$ with right endpoint the vertex $u_{x}$, i.e., $P^{\prime}=\mathcal{P}\left(u_{x} ; G_{u_{z}}^{r-1}\left(u_{p}, i, j\right)\right)$. 
In Case 3(II), Algorithm LP_Cocomparability computes (with the procedure append()) the value $w_{1}+1=\ell\left(u_{x} ; G_{u_{z}}^{r-1}\left(u_{p}, i, j\right)\right)+1$, for every vertex $u_{x} \in H_{\ell} \cap V\left(G_{u_{z}}^{r-1}\left(u_{p}, i, j\right)\right)$ such that $f\left(u_{t}\right) \leq \ell \leq j, x \neq t$, and $u_{x} u_{t} \notin E(G)$, and sets $\ell\left(u_{t} ; G_{u_{z}}^{r}\left(u_{p}, i, j\right)\right)$ to be equal to the maximum among these values. We next show that the algorithm correctly computes $\ell\left(u_{t} ; G_{u_{z}}^{r}\left(u_{p}, i, j\right)\right)=$ $\mathcal{L}\left(u_{t} ; G_{u_{z}}^{r}\left(u_{p}, i, j\right)\right)$ and $P\left(u_{t} ; G_{u_{z}}^{r}\left(u_{p}, i, j\right)\right)=\mathcal{P}\left(u_{t} ; G_{u_{z}}^{r}\left(u_{p}, i, j\right)\right)$.

3(II.a) Assume first that $u_{x} \notin L_{j}$, where $u_{x}$ is the right endpoint of $P^{\prime}$. Since by the induction hypothesis the algorithm correctly computes the values $\ell\left(u_{s} ; G_{u_{z}}^{r-1}\left(u_{p}, i, j\right)\right.$ for every vertex $u_{s} \in$ $G_{u_{z}}^{r-1}\left(u_{p}, i, j\right)$ such that $u_{s} \notin L_{j}$, it follows that Algorithm LP_Cocomparability computes, among other, the value $\ell\left(u_{x} ; G_{u_{z}}^{r-1}\left(u_{p}, i, j\right)\right)+1=\left|P^{\prime}\right|+1$, and sets $\ell\left(u_{t} ; G_{u_{z}}^{r}\left(u_{p}, i, j\right)\right)$ to be equal to $\left|P^{\prime}\right|+1=|P|$ which is equal to the length $\mathcal{L}\left(u_{t} ; G_{u_{z}}^{r}\left(u_{p}, i, j\right)\right)$ of a longest normal antipath $P$ of $G_{u_{z}}^{r}\left(u_{p}, i, j\right)$ with right endpoint the vertex $u_{t}$. Also, the corresponding computed antipath $P\left(u_{t} ; G_{u_{z}}^{r}\left(u_{p}, i, j\right)\right)=\left(P\left(u_{x} ; G_{u_{z}}^{r-1}\left(u_{p}, i, j\right)\right), u_{t}\right)$ is a longest normal antipath $\mathcal{P}\left(u_{t} ; G_{u_{z}}^{r}\left(u_{p}, i, j\right)\right)$ of $G_{u_{z}}^{r}\left(u_{p}, i, j\right)$ with right endpoint the vertex $u_{t}$.

3(II.b) Consider now the case where for any longest normal antipath $P=\left(u_{x^{\prime}}, \ldots, u_{x}, u_{t}\right)=\left(P^{\prime}, u_{t}\right)$ of $G_{u_{z}}^{r}\left(u_{p}, i, j\right)$ with right endpoint the vertex $u_{t}$ we have $u_{x} \in L_{j}$. Then $P^{\prime}$ is a longest normal antipath of $G_{u_{z}}^{r-1}\left(u_{p}, i, j\right)$ with right endpoint any vertex of $L_{j}$, i.e., $\left|P^{\prime}\right| \geq\left|P^{\prime \prime}\right|$ for any normal antipath $P^{\prime \prime}$ of $G_{u_{z}}^{r-1}\left(u_{p}, i, j\right)$ with right endpoint a vertex of $L_{j}$. Let $u_{x}$ be the rightmost vertex of $L_{j}^{r-1}\left(u_{z}\right)$ for which such an antipath $P^{\prime}$ exists. Since Algorithm LP_Cocomparability computes (with the procedure append()) the value $w_{1}+1=\ell\left(u_{x} ; G_{u_{z}}^{r-1}\left(u_{p}, i, j\right)\right)+1$ for every vertex $u_{x} \in L_{j}^{r-1}\left(u_{z}\right)$, and sets $\ell\left(u_{t} ; G_{u_{z}}^{r}\left(u_{p}, i, j\right)\right)$ to be equal to the maximum among these values, it follows that it suffices to show that there exists at least one vertex $u_{x} \in L_{j}^{r-1}\left(u_{z}\right)$ for which Algorithm LP_Cocomparability correctly computes the value $\ell\left(u_{x} ; G_{u_{z}}^{r-1}\left(u_{p}, i, j\right)\right)$ and sets it to be equal to $\left|P^{\prime}\right|=\mathcal{L}\left(u_{x} ; G_{u_{z}}^{r-1}\left(u_{p}, i, j\right)\right)$.

3(II.b.1) Consider first the case where $u_{x}$ is the last vertex of $L_{j}^{r-1}\left(u_{z}\right)$, i.e., there exists such a longest normal antipath $P^{\prime}$ of $G_{u_{z}}^{r-1}\left(u_{p}, i, j\right)$ with right endpoint a vertex of $L_{j}$, for which the right endpoint $u_{x}$ of $P^{\prime}$ is the last vertex of $L_{j}^{r-1}\left(u_{z}\right)$. Then by the induction hypothesis, Algorithm LP_Cocomparability correctly computes the length $\ell\left(u_{x} ; G_{u_{z}}^{r-1}\left(u_{p}, i, j\right)\right)$ of a longest normal antipath of $G_{u_{z}}^{r-1}\left(u_{p}, i, j\right)$ with right endpoint the vertex $u_{x}$. Therefore, in this case the last vertex $u_{x}$ of $L_{j}^{r-1}\left(u_{z}\right)$ is such a vertex for which $\ell\left(u_{x} ; G_{u_{z}}^{r-1}\left(u_{p}, i, j\right)\right)=\mathcal{L}\left(u_{x} ; G_{u_{z}}^{r-1}\left(u_{p}, i, j\right)\right)=\left|P^{\prime}\right|$; thus, Claim 1 holds.

3(II.b.2) Consider now the case where $u_{x}$ is not the last vertex of $L_{j}^{r-1}\left(u_{z}\right)$, i.e., $u_{x} \in L_{j}^{r-2}\left(u_{z}\right)$. Let $u_{q}$ be the last vertex of $L_{j}^{r-1}\left(u_{z}\right)$. Since $P^{\prime}$ is a longest normal antipath of $G_{u_{z}}^{r-1}\left(u_{p}, i, j\right)$ with right endpoint any vertex of $L_{j}$, it follows that $u_{q} \in V\left(P^{\prime}\right)$, since otherwise $\widetilde{P}=\left(P^{\prime}, u_{q}\right)$ is such a normal antipath longer than $P^{\prime}$. Let $P^{\prime}=\left(u_{x^{\prime}}, \ldots, u_{q^{\prime}}, u_{q}, u_{q^{\prime \prime}}, \ldots, u_{x}\right)=\left(P_{1}, u_{q}, P_{2}\right)$. We now prove that $u_{q^{\prime}}<_{\sigma} u_{q}$. Since $u_{q} \in L_{j}$, it follows that $u_{q^{\prime}} \leq_{\sigma} u_{q}$. Assume that $u_{q^{\prime}}={ }_{\sigma} u_{q}$. Then using Lemma 2.4 and Definition 2.2, we can easily prove that $u_{s}={ }_{\sigma} u_{q}$ for every vertex $u_{s} \in V\left(P_{2}\right)$. Thus, $P^{\prime \prime}=\left(P_{1}, u_{q^{\prime \prime}}, \ldots, u_{x}, u_{q}\right)$ is a normal antipath such that $V\left(P^{\prime \prime}\right)=V\left(P^{\prime}\right)$ with right endpoint the vertex $u_{q}$ which appears after $u_{x}$ in $L_{j}^{r-1}\left(u_{z}\right)$; this is a contradiction to our choice of $u_{x}$. Therefore, we have proved that $u_{q^{\prime}}<_{\sigma} u_{q}$. Therefore, using the same arguments as in Claim 2 and the property that $u_{q^{\prime}}<_{\sigma} u_{q}$, we can prove that $u_{q^{\prime}}<_{\sigma} u_{s}$ and $u_{q^{\prime}} u_{s} \in E(G)$, for every vertex $u_{s} \in V\left(P_{2}\right)$.

Since $u_{q^{\prime}} u_{q} \notin E(G)$ and $u_{q^{\prime}}<_{\sigma} u_{q}$, we assume that $u_{q^{\prime}} \in H_{\ell}, f\left(u_{q}\right) \leq \ell \leq j-1$. Let $\mathcal{H}_{2}$ be the subgraph of $G_{u_{z}}^{r-1}\left(u_{p}, i, j\right)$ induced by $V\left(\mathcal{H}_{2}\right)=V\left(G_{u_{z}}^{r-2}\left(u_{q^{\prime}}, \ell+1, j\right)\right)$ and let $\mathcal{H}_{1}$ be the subgraph of $G_{u_{z}}^{r-1}\left(u_{p}, i, j\right)$ induced by $V\left(\mathcal{H}_{1}\right)=V\left(G_{u_{z}}^{r-2}\left(u_{p}, i, j\right)\right) \backslash V\left(G_{u_{z}}^{r-2}\left(u_{q^{\prime}}, \ell+1, j\right)\right)$. Using the same arguments as in Claim 3 and the property that $u_{q^{\prime}}<_{\sigma} u_{q}$, we can show that every vertex of $P_{2}$ belongs to $\mathcal{H}_{2}$, and also that every vertex of $P_{1}$ belongs to $\mathcal{H}_{1}$. Therefore, we have that $V\left(P_{1}\right) \subseteq V\left(\mathcal{H}_{1}\right), V\left(P_{2}\right) \subseteq V\left(\mathcal{H}_{2}\right)$, and $V\left(\mathcal{H}_{1}\right) \cap V\left(\mathcal{H}_{2}\right)=\emptyset$; thus, $V\left(P_{1}\right) \cap V\left(P_{2}\right)=\emptyset$. Finally, using the same arguments as in Case 2(I) we can obtain that $P_{1}$ is a longest normal antipath of $G_{u_{z}}^{r-2}\left(u_{p}, i, j\right)$ with right endpoint the vertex $u_{q^{\prime}}$, i.e., $\left|P_{1}\right|=\mathcal{L}\left(u_{q^{\prime}} ; G_{u_{z}}^{r-2}\left(u_{p}, i, j\right)\right)$, 
and $P_{2}$ is a longest normal antipath of $G_{u_{z}}^{r-2}\left(u_{q^{\prime}}, \ell+1, j\right)$ with right endpoint the vertex $u_{x}$, i.e., $\left|P_{2}\right|=\mathcal{L}\left(u_{x} ; G_{u_{z}}^{r-2}\left(u_{q^{\prime}}, \ell+1, j\right)\right)$.

Since $u_{q^{\prime}}<_{\sigma} u_{q}$, it follows that $u_{q^{\prime}} \notin L_{j}$. Therefore, from the induction hypothesis Algorithm LP_Cocomparability correctly computes the length $\ell\left(u_{q^{\prime}} ; G_{u_{z}}^{r-2}\left(u_{p}, i, j\right)\right)=\left|P_{1}\right|$. Now it is left to show that the value $\ell\left(u_{x} ; G_{u_{z}}^{r-2}\left(u_{q^{\prime}}, \ell+1, j\right)\right)=\left|P_{2}\right|$ computed by the algorithm is equal to $\mathcal{L}\left(u_{x} ; G_{u_{z}}^{r-2}\left(u_{q^{\prime}}, \ell+1, j\right)\right)$. Observe that now $P_{2}$ is a longest normal antipath of $G_{u_{z}}^{r-2}\left(u_{q^{\prime}}, \ell+1, j\right)$ with right endpoint any vertex of $L_{j}^{r-2}\left(u_{z}\right)$ and, actually, $u_{x}$ is the rightmost vertex of $L_{j}^{r-2}\left(u_{z}\right)$ for which such an antipath $P_{2}$ exists, otherwise we come to a contradiction to the choice of $P^{\prime}$ (note that $u_{q}$ is connected with an antiedge to every vertex of $\left.P_{2}\right)$. If $u_{x}$ is the last vertex of $L_{j}^{r-2}\left(u_{z}\right)$ then, similarly to the above, by the induction hypothesis the algorithm correctly computes the value $\ell\left(u_{x} ; G_{u_{z}}^{r-2}\left(u_{q^{\prime}}, \ell+1, j\right)\right)$. If $u_{x}$ is not the last vertex of $L_{j}^{r-2}\left(u_{z}\right)$, then we repeat the above same procedure of Case 3(II.b.2), where now by $u_{q}$ we denote the last vertex of $L_{j}^{r-2}\left(u_{z}\right)$.

We repeat the above procedure until $u_{x}$ is the last vertex of the ordering $L_{j}^{r^{\prime}}\left(u_{z}\right), 1 \leq r^{\prime} \leq r-2$, i.e., we repeat the above procedure $\left(r-2-r^{\prime}\right)$ times in total. Let $P^{\prime}=\left(P_{1}, P_{2}, \ldots, P_{r-2-r^{\prime}+1}\right)$ be the longest normal antipath $P^{\prime}$ of $G_{u_{z}}^{r-1}\left(u_{p}, i, j\right)$ with right endpoint the vertex $u_{x}$, such that at the $s^{t h}$ iteration of the above procedure we prove that Algorithm LP_Cocomparability correctly computes the antipath $P_{s}, 1 \leq s \leq r-2-r^{\prime}$. Finally, at the $\left(r-2-r^{\prime}\right)^{t h}$ iteration we also obtain by the induction hypothesis that the algorithm correctly computes the antipath $P_{r-2-r^{\prime}+1}$ which has $u_{x}$ as its right endpoint, since at that iteration $u_{x}$ is the last vertex of the ordering $L_{j}^{r^{\prime}}\left(u_{z}\right)$. Concluding, Algorithm LP_Cocomparability correctly computes the length $\ell\left(u_{x} ; G_{u_{z}}^{r-1}\left(u_{p}, i, j\right)\right)=\left|P^{\prime}\right|$ and, thus, the length $\ell\left(u_{t} ; G_{u_{z}}^{r}\left(u_{p}, i, j\right)\right)=\left|P^{\prime}\right|+1=|P|$; the algorithm also correctly computes the corresponding antipaths.

Concluding, we have proved that Claim 1 holds for the subgraph $G_{u_{z}}^{r}\left(u_{p}, i, j\right)$ of $G$, where $1 \leq r \leq\left|L_{j}\right| ; \quad Q E D$ of Claim 1 .

Let $P$ be a longest antipath of $G$ such that $|P| \geq 2$. From Lemma 2.6 we may assume that $P$ is a longest normal antipath of $G$ and let $u_{y}$ be its right endpoint. Also, $P$ belongs to the graph $G \backslash\left\{u_{0}\right\}$. Since $G\left(u_{0}, 1, k\right)=G \backslash\left\{u_{0}\right\}$ and since Algorithm LP_Cocomparability computes the maximum among the lengths $\left\{\ell\left(u_{y} ; G\left(u_{0}, 1, k\right)\right): u_{y} \in V\left(G\left(u_{0}, 1, k\right)\right)\right\}$ and the corresponding antipath $P^{\prime}$, from Lemma 4.3 we obtain that $\left|P^{\prime}\right|=|P|$. Therefore, we obtain the following.

Theorem 4.1 Algorithm LP_Cocomparability computes a longest path of a cocomparability graph in polynomial time.

\subsection{Time Complexity}

Let $G$ be a comparability graph on $|V(G)|=n$ vertices and $|E(G)|=m$ edges. Given a Hasse diagram of $G$, the time complexity of our algorithm is as follows.

Algorithm LP_Cocomparability executes the subroutine process() for every induced subgraph $G_{u_{z}}^{r}\left(u_{p}, i, j\right)$ of $G$. In particular, the procedure process() contains two procedures namely bridge() and append(). The execution of the procedure bridge() for the subgraph $G_{u_{z}}^{r}\left(u_{p}, i, j\right)$ takes $O\left(n^{2}\right)$ time, due to the $O\left(n^{2}\right)$ pairs of antineighbors $u_{x}$ and $u_{y}$ of the vertex $u_{t}$ in the graph $G_{u_{z}}^{r}\left(u_{p}, i, j\right)$. The execution of the procedure append() for the subgraph $G_{u_{z}}^{r}\left(u_{p}, i, j\right)$ takes $O(n)$ time, due to the $O(n)$ antineighbors $u_{x}$ of the vertex $u_{t}$ in the graph $G_{u_{z}}^{r}\left(u_{p}, i, j\right)$. Therefore, the execution of the procedure process () for the subgraph $G_{u_{z}}^{r}\left(u_{p}, i, j\right)$ takes $O\left(n^{2}\right)$ time.

Additionally, process () is executed at most once for each subgraph $G_{u_{z}}^{r}\left(u_{p}, i, j\right)$ of $G$. Since $1 \leq i \leq j \leq k, u_{p} \in H_{i-1}, u_{z} \in L_{j}$, and $1 \leq r \leq\left|L_{j}\right|$, it follows that there exist $O\left(n^{5}\right)$ such subgraphs $G_{u_{z}}^{r}\left(u_{p}, i, j\right)$ of $G$. Thus, Algorithm LP_Cocomparability takes $O\left(n^{7}\right)$ time.

In order to compute the length of a longest antipath, we need to store one value for every vertex $u_{y}$ of $G_{u_{z}}^{r}\left(u_{p}, i, j\right)$, for every induced subgraph $G_{u_{z}}^{r}\left(u_{p}, i, j\right)$ of $G$. Thus, since there are in 
total $O\left(n^{5}\right)$ such subgraphs $G_{u_{z}}^{r}\left(u_{p}, i, j\right)$, and since each one has at most $O(n)$ vertices, we can compute the length of a longest antipath in $O\left(n^{6}\right)$ space. Furthermore, in order to compute and report a longest antipath, instead of its length only, we have to store an antipath of at most $n$ vertices for each one of the $O\left(n^{6}\right)$ computed values. Therefore, the space complexity of Algorithm LP_Cocomparability is $O\left(n^{7}\right)$.

\section{Concluding Remarks}

In this work we presented a polynomial-time algorithm for solving the longest path problem on cocomparability graphs, resolving thus the open question on the complexity status of the problem on cocomparability and, thus, on permutation graphs. We also help to shed some light on the borderline between $\mathrm{P}$ and NP, since the longest path problem is known to be NP-complete on comparability graphs and quasi-parity graphs, while it polynomial on permutation and cocomparability graphs.

It would be interesting to study the complexity of the longest path problem on distancehereditary and bipartite distance-hereditary graphs, since they admit polynomial solutions for the Hamiltonian path problem, and also since the longest path problem has been proved to be NPcomplete on chordal bipartite graphs, HHD-free graphs, and parity graphs, while it is polynomial on ptolemaic graphs and trees. Additionally, the same holds for the classes of convex and biconvex graphs, since the longest path problem has been proved to be NP-complete on chordal bipartite graphs and polynomial on bipartite permutation graphs.

\section{References}

[1] S.R. Arikati and C. Pandu Rangan, Linear algorithm for optimal path cover problem on interval graphs, Inform. Proc. Lett. 35 (1990) 149-153.

[2] K. Asdre and S.D. Nikolopoulos, The 1-fixed-endpoint path cover problem is polynomial on interval graphs, Algorithmica, 58 (2010) 679-710.

[3] A.A. Bertossi, Finding Hamiltonian circuits in proper interval graphs, Inform. Proc. Lett. 17 (1983) 97-101.

[4] A. Brandstädt, V.B. Le and J.P. Spinrad, Graph Classes: A Survey, SIAM, Philadelphia, PA, 1999.

[5] R. Bulterman, F. van der Sommen, G. Zwaan, T. Verhoeff, A. van Gasteren, and W. Feijen, On computing a longest path in a tree, Inform. Proc. Lett. 81 (2002) 93-96.

[6] M.S. Chang, S.L. Peng, and J.L. Liaw, Deferred-query: An efficient approach for some problems on interval graphs, Networks 34 (1999) 1-10.

[7] P. Damaschke, The Hamiltonian circuit problem for circle graphs is NP-complete, Inform. Proc. Lett. 32 (1989) $1-2$.

[8] P. Damaschke, Paths in interval graphs and circular arc graphs. Discrete Math. 112 (1993) 49-64.

[9] P. Damaschke, J.S. Deogun, D. Kratsch, and G. Steiner, Finding Hamiltonian paths in cocomparability graphs using the bump number algorithm, Order 8 (1992) 383-391.

[10] J.S. Deogun and C. Riedesel, Hamiltonian cycles in permutation graphs, J. Comb. Math. and Comb. Computing 27 (1998) 161-200.

[11] J.S. Deogun and G. Steiner, Polynomial algorithms for hamiltonian cycle in cocomparability graphs, SIAM J. Computing 23 (1994) 520-552.

[12] T. Feder and R. Motwani, Finding large cycles in Hamiltonian graphs, Discrete Appl. Math. 158 (2010) 882-893.

[13] H.N. Gabow, Finding paths and cycles of superpolylogarithmic length, SIAM J. Computing 36 (2007) 1648-1671. 
[14] H.N. Gabow and S. Nie, Finding long paths, cycles and circuits, Proc. of the 19th annual International Symp. on Algorithms and Computation (ISAAC), LNCS 5369 (2008) 752-763.

[15] M.R. Garey and D.S. Johnson, Computers and Intractability: A Guide to the Theory of NPcompleteness, W.H. Freeman, New York, 1979.

[16] M.R. Garey, D.S. Johnson, and R.E. Tarjan, The planar Hamiltonian circuit problem is NP-complete, SIAM J. Computing 5 (1976) 704-714.

[17] M.C. Golumbic, Algorithmic Graph Theory and Perfect Graphs (Annals of Discrete Mathematics, Vol. 57), North-Holland Publishing Co., Amsterdam, The Netherlands, 2004.

[18] M. Habib, R.H. Mörhing, and G. Steiner, Computing the bump number is easy, Order 5 (1988) $107-129$.

[19] K. Ioannidou, G.B. Mertzios, and S.D. Nikolopoulos, The longest path problem has a polynomial solution on interval graphs, Algorithmica, doi: 10.1007/s00453-010-9411-3.

[20] A. Itai, C.H. Papadimitriou, and J.L. Szwarcfiter, Hamiltonian paths in grid graphs, SIAM J. Computing 11 (1982) 676-686.

[21] D. Karger, R. Motwani, and G.D.S. Ramkumar, On approximating the longest path in a graph, Algorithmica 18 (1997) 82-98.

[22] T.A. McKee and F.R. McMorris, Topics in Intersection Graph Theory, Society for Industrial and Applied Mathematics, Philadelphia, 1999.

[23] H. Müller, Hamiltonian circuits in chordal bipartite graphs, Discrete Math. 156 (1996) 291-298.

[24] G. Narasimhan, A note on the Hamiltonian circuit problem on directed path graphs, Inform. Proc. Lett. 32 (1989) 167-170.

[25] Y. Takahara, S. Teramoto, and R. Uehara, Longest path problems on ptolemaic graphs, IEICE Trans. Inf. and Syst. 91-D (2008) 170-177.

[26] R. Uehara, Simple geometrical intersection graphs, Proc. of the 2nd annual Workshop on Algorithms and Computation (WALCOM'08), LNCS 4921 (2004) 25-33.

[27] R. Uehara and Y. Uno, Efficient algorithms for the longest path problem, Proc. of the 15th annual International Symp. on Algorithms and Computation (ISAAC), LNCS 3341 (2004) 871-883.

[28] R. Uehara and G. Valiente, Linear structure of bipartite permutation graphs and the longest path problem, Inform. Proc. Lett. 103 (2007) 71-77.

[29] S. Vishwanathan, An approximation algorithm for finding a long path in Hamiltonian graphs, Proc. of the 11th annual ACM-SIAM Symp. on Discrete Algorithms (SODA), ACM (2000) 680-685.

[30] Z. Zhang, and H. Li, Algorithms for long paths in graphs, Theoret. Comput. Sci. 377 (2007) 25-34. 